\title{
Temperature and rate dependent thermomechanical modeling of shape memory polymers with physics based phase evolution law
}

\author{
Qianxi Yang ${ }^{1}$ and Guoqiang $\mathrm{Li}^{1,2^{*}}$ \\ ${ }^{1}$ Department of Mechanical \& Industrial Engineering, Louisiana State University, \\ Baton Rouge, LA 70803, USA \\ ${ }^{2}$ Department of Mechanical Engineering, Southern University, Baton Rouge, LA \\ 70813, USA \\ *Correspondence author: Tel.: 001-225-578-5302; Fax: 001-225-578-5924; E- \\ mail: lguoqi1@1su.edu
}

\begin{abstract}
:
The thermomechanical constitutive models are critical for shape memory polymers (SMPs) in analyzing their shape memory behaviors under different conditions. Recently, the phase evolution modeling approach proposed by Liu et al. (Liu et al., 2006) has been extended and widely used due to its simplicity and generality. However, the lack of physical explanation has become its major limitation. In this study, a phase evolution law was proposed from the physics perspective. The shape memory polymer was then treated as a frozen-phase matrix with active-phase inclusions embedded in it and the Mori-Tanaka approach was used to predict the effective mechanical properties. The theoretical predictions were compared with available experimental results and reasonable agreement was found. The influence of the intrinsic material structures and the thermomechanical conditions on the shape memory behavior were evaluated by parametric studies.
\end{abstract}

Keywords: Shape memory polymer; Constitutive modeling; Phase evolution law;

\section{Introduction}

Shape memory polymer (SMP) is a class of smart materials that can be deformed and fixed in a temporary shape at a certain condition and recovers the original shape when exposed to a suitable stimulus (Dietsch and Tong, 2007; Hu et al., 2012; Huang et al., 2010; Lendlein and Kelch, 2002; Leng et al., 2011; Leng et al., 2009; Meng and Li, 2013; Mather et al., 2009; Ratna and Karger-Kocsis, 2008; Zhao et al., 2015), such as temperature (Behl and Lendlein, 2007; Chung et al., 2008; Liu et al., 2007; Xie, 2010; Yu et al., 2012), light (Lendlein et al., 2005; Scott et al., 2005), magnetic field (Buckley et al., 2006; Schmidt, 2006) or humidity (Chae Jung et al., 2006; Huang et al., 2005). The term "shape-memory" was first proposed by Vernon in 1941 (Vernon and Vernon, 1941). Although most polymers can exhibit some degree of shape memory behavior, the importance of SMPs was not recognized until the 1960s, when cross-linked polyethylene (PE) were used as shrinkage tape to apply pressure (Hitov et al., 1964). SMPs have many advantages over shape memory alloys and ceramics, such as low manufacturing cost, easy processing and most significantly, high shape recovery strain (maximum recovery strain can be over $800 \%$ (Zhao et al., 2015). Based on these unique features, SMPs have emerged as one of the key functional materials in both academia and industry in recent years. 
Of all types of SMPs, thermally triggered SMP is the first and the most widely used one. When studying the thermally responsive SMPs, programming step, that is, thermomechanical training, is the essential process to give the material "memory". Typically, the programming process starts with a deformation of the SMP at a temperature above the transition temperature, for example, glass transition $T_{g}$ for amorphous SMPs or melting transition $T_{m}$ for crystalline or semi-crystalline SMPs. For ease of discussion, however, we hereafter refer only to glass transition $T_{g}$. While maintaining the shape (strain) or stress, the temperature is lowered to below $T_{g}$. With the subsequent removal of the applied load, a temporary shape is created and fixed. This completes the programming process. It is noted that programming does not necessarily need the temperature event. Programming can also be conducted at glassy state, which is termed as cold-programming, as long as the SMP can be stressed to beyond yielding (Li, 2014; Li et al., 2013; Li and Zhang, 2013; Li and Shojaei, 2012; Li and Xu, 2011; Xu and Li, 2011). The recovery process, depending on the application where the recovery strain or stress is important, can be either under free or constrained boundary conditions, respectively. During the free recovery, SMP is heated to a temperature above $T_{g}$ under zero stress and the change in strain is measured. On the other hand, during the stress recovery, deformation is not allowed while the polymer is heated to above $T_{g}$ and the stress is measured. Partially constrained shape recovery by applying a certain stress to the SMP during shape recovery is also frequently used (Li and Nettles, 2010). The shape memory performance of thermally triggered SMPs depends on their complex interactions in polymer structures and morphologies (e.g., the crosslink density, molecular length), the programming conditions (e.g., programming temperature, cooling rate, pre-strain) and recovery conditions (e.g., recovery temperature, heating rate, boundary condition). This offers substantial potentials and considerable opportunities for designing SMPs. In fact, in recent years, research on designing thermally triggered SMPs has developed tremendously. Apart from the classical one-way SMPs, two-way, triple, multiple-shape SMPs, and even temperaturememory effects have been widely investigated in SMPs. A review on the recent shape memory functionality design can be found in (Hu et al., 2012; Zhao et al., 2015).

In engineering applications of SMPs, constitutive models are highly desired to provide a fundamental design tool. However, constitutive modeling is very involved because of the inherent complexity of the shape memory behavior. While considerable experimental investigations into SMPs have been reported in the literature, the number of published works dedicated to constitutive modeling constitutes only a small portion of the vast volume of published literatures. Earlier methods (Buckley et al., 2007; Lin and Chen, 1999; Tobushi et al., 1997; Tobushi et al., 2001) used rheological models consisting of springs, dashpots, and frictional elements to interpret the thermomechanical behavior of SMPs. Such distinctly simple models have recently been expanded into three dimensional finite deformation model and reasonable results were obtained (Balogun and Mo, 2014; Ghosh and Srinivasa, 2014). Diani et al. (Diani et al., 2006) developed a three dimensional thermoviscoelastic constitutive model based on a physical understanding of the material behavior. This thermodynamically motivated model is able to capture some critical features of the shape memory behavior, however, it does not predict the details of the response in the thermomechanical cycle. To further capture the time- and temperature-dependent feature of amorphous SMPs, a thermoviscoelastic model that incorporated structural relaxation and stress activated viscoplastic flow in the glassy region was proposed from a 
microscopic point of view (Nguyen et al., 2008). The model was able to predict key features of the stress hysteresis of the constrained recovery response, including the peak stress and associated temperature. Subsequently, Chen and Nguyen (Chen and Nguyen, 2011) performed a parameteric study of Nguyen et al.'s model (Nguyen et al., 2008), and drew the conclusion that the recovery behavior was insensitive to the parameters for viscoplastic flow of materials programmed above $T_{g}$. However, $\mathrm{Li}$ and $\mathrm{Xu}$ (Li and $\mathrm{Xu}, 2011$ ) argued that the viscoplastic flow plays an important role in the transition between equilibrium and non-equilibrium configuration of SMPs when cold-programmed (program in the glassy state), and a thermoviscoelastic-thermoviscoplastic constitutive model was then developed on top of that to predict the nonlinear shape memory behavior of the SMP trained below $T_{g}$. To further improve the model prediction of time-dependent shape memory behavior, more sophisticated models that contained multiple relaxation times (Buckley et al., 2007; Chen and Nguyen, 2011; Diani et al., 2012; Ge et al., 2012; Westbrook et al., 2011; Xiao et al., 2013; Yu et al., 2012) were used to describe the broad distribution of stress and structural relaxation times typically displayed by thermoset polymers. Recently, the established 3D multi-branch model (Westbrook et al., 2011) has been applied successfully to predict various time-and temperature- dependent shape memory behavior (Ge et al., 2013; Ge et al., 2014; Yu et al., 2014). In addition to this dominated thermoviscoelastic approach, in recent years, SMP modeling methods have even incorporated molecular dynamic simulation (Diani and Gall, 2007), quantum mechanics (Zhang et al., 2010), multi-scale modeling (Shojaei and $\mathrm{Li}, 2013$ ), and statistical mechanics (Shojaei and $\mathrm{Li}$, 2014), etc. Of all these methods, the phase evolution approach, first proposed by Liu et al. (Liu et al., 2006) and further developed by other researchers (Baghani et al., 2012; Chen and Lagoudas, 2008a, b; Gilormini and Diani, 2012; Guo et al., 2015; Kafka, 2008; Kazakevičiūtè-Makovska et al., 2012; Kim et al., 2010; Long et al., 2010; Pieczyska et al., 2015; Qi et al., 2008; Reese et al., 2010; Scalet et al., 2015; Volk et al., 2011; Wang et al., 2009; $\mathrm{Xu}$ and $\mathrm{Li}, 2010$ ) has become another widely used modeling approach due to its ease of application in design.

The phase evolution approach was originally developed for shape memory alloys (SMAs) to describe their pseudo-elastic thermomechanical response, stress induced phase transformation phenomena, and hence the shape memory behavior (Auricchio et al., 2007; Dachkovski and Böhm, 2004; Hassan et al., 2008; Levitas and Ozsoy, 2009a, b; Moumni et al., 2008; Müller and Bruhns, 2006; Popov and Lagoudas, 2007; Reese and Christ, 2008; Thamburaja and Ekambaram, 2007; Thamburaja and Nikabdullah, 2009; Wang et al., 2008). Unlike in SMAs, however, the concept of "phase" in SMPs may not necessarily be physically based, but rather phenomenologically based. In other words, in amorphous SMPs, the physical regions with different material properties and the boundaries between them may not be measurable. In the first SMP phase evolution model (Liu et al., 2006), a one-dimensional, small strain and rate independent constitutive model were developed by considering the SMP system as a two-phase composite consitsting of an active and a frozen phase. In the active state, the deformation mainly causes a change in entropy and the resulting stress can be determined by the entropic rubber theory. In the frozen phase, the entropic changes are "locked" owing to a decreased mobility of individual polymer chains and the resulting stress is primarily due to changes in the internal energy. Therefore, the entropic stress generated in the active state is frozen, or stored, and can only be released by heating above the transition temperature to reactivate the entropic changes. Based on the 
work by Liu et al. (Liu et al., 2006), Chen and Lagoudas (Chen and Lagoudas, 2008a, b) extended the model to a three-dimensional framework and this model was used by Volk et al. (Volk et al., 2011) to examine the free and constraint recovery behavior of shape memory polyurethane (SMPU). Kim et al. (Kim et al., 2010) developed a three-phase phenomenological model (one hard segment phase and two (active and frozen) soft segment phases) to provide more proper prediction for the deformation behavior of SMPUs. In Wang et al.'s work (Wang et al., 2009), parameters related to heating rate and hysteresis were introduced. Qi et al. (Qi et al., 2008) developed a three dimensional finite deformation model for thermomechanical behavior of SMPs, based on the evolution of the deformation energy from an entropy- to an enthalpy-based state. Reese et al. (Reese et al., 2010) formulated the model in both macromechanical and micromechanical frameworks and the thermomechanial coupling was taken into account. Baghani et al. (Baghani et al., 2012) presented a three-dimensional phenomenological model under time-dependent multiaxial thermo-mechanical loadings in the small strain regime. In the work by (Gilormini and Diani, 2012), one point that was left open by Chen and Lagoudas (Chen and Lagoudas, 2008a, b) about combining the model of Liu et al. (Liu et al., 2006) with homogenization models that would be more accurate than the uniform stress assumption was addressed. Most recently, a constituve model for SMP with more physically based phase evolution law has been proposed by Guo et al. (Guo et al., 2015). Once the SMP system is considered as a two-phase composite, its effective elastic properties can be determined by various well developed composite material theories (Hill, 1965; Hori and Nemat-Nasser, 1993; Hu and Weng, 2000; Ju and Chen, 1994; Li and Wang, 2005; Mori and Tanaka, 1973; Peng et al., 2009).

The concept of phase transition approach is not only very straightforward, it can also be applied to a wide variety of SMP materials with different transition mechanisms. It can find solid physical base in semicrystalline SMPs (Scalet et al., 2015). Westbrook et al. (Westbrook et al., 2010) successfully applied the phase-based modeling approach to the one-way and two-way shape memory effect in semicrystalline poly(cyclooctene) (PCO) SMP. Long et al. (Long et al., 2009) recently has developed a model for photo-activated shape memory elastomers that treats the material as a mixture of two evolving networks, the original network and the photo-stimulated reformed network.

In the framework of phase transition modeling, the formulation of a suitable phase evolution law, in other words, the frozen phase volume fraction, $\phi(T)$ is essential. A variety of frozen phase volume fraction functions have been proposed by researchers and some typical functions are listed in Table 1. As can be seen from Table 1, although these evolution laws possess simple form, the parameters in the equations cannot be explicitly related to the inherent material properties and structures (cross-linking density, molecular length, etc.), nor the thermomechanical conditions (heating rate, pre-strain, etc.). Up to now, the lack of physical explanation has become the main limitation of the phase transition approach.

This present work aims at developing a physics-based phase evolution law so that the phase transition approach can be used more widely with confidence in the future. In the first section of this paper, a brief review on the theory of glass transition is given, which is important to phase evolution for amorphous polymers. Then, a general phase evolution law for amorphous SMPs is elucidated and the frozen phase volume fraction function is formulated, based on the existing physical laws. In the section that follows, the constitutive 
relations for the amorphous SMPs are developed using the established evolution law, together with the classical composite materials theories. Our model is then applied to the SMP system used by Liu et al. (Liu et al., 2006) and the modeling results are verified with the experimental data. Finally, a parametric study is performed to evaluate the influence of several key parameters on the phase evolution law.

Table 1

Frozen phase volume fraction functions proposed in existing literatures.

\begin{tabular}{ll}
\hline Frozen phase volume fraction $\phi(T)$ & Reference \\
$\phi(T)=1-\frac{1}{1+c_{f}\left(T_{h}-T\right)^{n}}$ & (Liu et al., 2006) \\
$\phi(T)=\frac{1}{1+\exp \left(-\frac{\left(T-T_{t}\right)}{T}\right)}$ & (Qi et al., 2008) \\
$\phi(T)=\alpha \exp \left(-\left(\frac{T_{t}}{T}\right)^{m} \beta^{-n}\right)$ & (Wang et al., 2009) \\
$\phi(T)=\frac{1}{1+\exp \left(\frac{2 w}{T-T_{t}}\right)}$ & (Reese et al., 2010) \\
$\phi(T)=\frac{b-\tanh ((T-A) / B)}{b-a}$ & (Volk et al., 2011) \\
$\phi(T)=\left(1-\left(\frac{T-T_{\min }}{T_{\max }-T_{\min }}\right)^{m}\right)^{n}$ & (Gilormini and \\
$\phi(T)=\int_{T_{s}}^{T} \frac{1}{S \sqrt{2 \pi}} \exp \left(-\frac{\left(T-T_{g}\right)}{2 S^{2}}\right) d T$ & Diani, 2012) \\
\hline
\end{tabular}

\section{Phase evolution law}

\subsection{Perspectives on glass transition}

For amorphous SMPs, shape recovery takes place near the glass transition region. Typically, there are two explanations for the driving force in the shape recovery. One is entropy driven (Behl and Lendlein, 2007), and the other is structural relaxation driven (Li and $\mathrm{Xu}, 2011$; Nguyen et al., 2008) when SMPs evolve through the glass transition region. In the entropy driven theory, the driving force for shape recovery is the conformational entropy of the molecular chains in terms of micro-Brownian thermal motion. Thermodynamically, the molecular chains experience a change from an ordered but temporary configuration to its original random and coiled configuration during the shape recovery process. Since this process is accompanied by an increase in entropy, it is thermodynamically an autonomous process. In the structural relaxation driven theory, it is suggested that the dramatic change in the temperature dependence of the molecular chain mobility underpins the thermally activated shape memory phenomena of SMPs. The fact that the structure relaxes instantaneously to equilibrium at temperature above $T_{g}$ but responds sluggishly at temperature below $T_{g}$ suggests that, reheating programmed SMPs to above $T_{g}$ reduces the viscosity, restores the mobility and allows the structure to relax to its equilibrium configuration, which leads to shape recovery.

Because of this, understanding the glass transition is the key to elucidating the shape recovery behavior of amorphous SMPs. Despite many decades of intensive research, the glass transition phenomenon is still one of the most challenging topics in polymer science. The glass transition was first explained from the thermodynamic aspect due to the development of calorimetric measurement techniques (Debenedetti, 1996; Glotzer, 2000). 
In order to explain the questions and paradox identified by Kauzmann (Kauzmann, 1948), glass transition studies were deviated into kinetic direction, where the kinetic properties, such as shear viscosity, mean shear-stress and thermal relaxation time, and the selfdiffusion constant were discussed. Among the numerous approaches successfully accounting for the steep increase in the relaxation time of the glass transition with decreasing temperature, the most employed is the so-called Vogel-Fulcher-Tammann (VFT) equation (Fulcher, 1925; Tammann and Hesse, 1926; Vogel, 1921) and the subsequent GD (Gibbs and DiMarzio)-AG (Adam and Gibbs) theory (Adam and Gibbs, 1965; Gibbs and DiMarzio, 1958). In the last decades, the research in glass transition has seen an explosive growth, thanks to the remarkable development in computation power and experimental techniques. New conceptual tools have been introduced (such as energy landscape (Wales, 2004)), new theoretical directions have been pioneered (for example, the random energy models (Sasai, 2003)), and new experimental results have been established (for instance, the spatially heterogeneous dynamics (Andersen, 2005; Ediger, 2000; Glotzer, 2000; Richert, 2002)). These developments provide a solid physical background for our phase evolution law.

\subsection{Formulation of phase evolution law}

\subsubsection{Facts and assumptions}

One of the recently developed theories on glass transition, the dynamic heterogeneity suggested the co-existence of spatially separated regions of various sizes in amorphous polymer system, whose relaxation dynamics can differ from each other by a factor of up to five orders of magnitude (Ediger, 2000). Therefore, when observed at an intermediate time scale, the whole system can then be classified into immobile and mobile domains, which correspond to frozen and active phases, respectively, in shape memory polymer studies. In particular, in the immobile domains, or the frozen phase, the molecular chains are restricted by the secondary interaction and cannot move cooperatively; while in the mobile domains, or the active phase, the molecular chains can move freely because the barriers between chains have been broken by the external work. The active phase thus has higher energy compared to the frozen phase and work must be done to overcome this barrier when the frozen phase transforms into the active phase. As such, the transformation from the frozen phase to the active phase bears strong resemblance to the energy level transition. Having noticed this, two requirements must be satisfied to ensure the accomplishment of the transition for each domain: first, the size of the domain must reach the critical value so that the transition is thermodynamically stable upon perturbation (Liu et al., 2013); second, based on the energy landscape theory (Wales, 2003), sufficient observation time should be given, so that the transition can take place with high probability (Gupta and Mauro, 2007).

In the next section, we will formulate the evolution law based on the above facts and the following assumptions for the sake of simplicity: (1) the dynamic heterogeneous domains are spheres with radius $r$ that obey the quasi-Gaussian spatial distribution; (2) the hysteresis in glass transition is neglected here, such that the frozen phase volume fraction functions are the same upon heating and cooling when other conditions are maintained. 


\subsubsection{Evolution law formulation}

As stated in section 2.2.1, when a frozen domain is transformed into an active domain, work must be done to overcome the energy barrier. This energy barrier has two resources, one is the bulk free energy difference between the frozen and active domains, and the other is the interfacial energy introduced by the interface between the frozen and active domains. During transformation, a spherical frozen domain with radius $r$ evolves into an active domain (see Fig. 1(a)) and the total free energy change caused by the replacement of the active phase is:

$$
\Delta G(r)=\frac{4}{3} \pi r^{3} G_{a}+\Gamma-W-\frac{4}{3} \pi r^{3} G_{f}=\frac{4}{3} \pi r^{3}\left(\Delta G_{B}-\Delta G_{W}\right)+4 \pi r^{2} \gamma
$$

where $G_{a}$ and $G_{f}$ are the free energy per unit volume for the active and frozen phase at a certain temperature, respectively; $\Gamma$ is the surface energy between the two phases; $W$ is the external driving energy (due to heat, stress, light, humidity, etc.); $\Delta G_{B}$ is the difference of the bulk free energy between a unit frozen and active domain, i.e., $\Delta G_{B}=G_{a}-G_{f} ; \Delta G_{W}$ is the free energy change per unit volume by the external work; and $\gamma$ is the surface energy density which is assumed to be constant. The total free energy change as a function of radius $r$ is sketched in Fig. 1(b). As can be seen from the figure, there exists a critical radius $r_{c}$ (when $\Delta G$ reaches the maximum value $\Delta G_{c}$ ). Only when the domain is large enough (larger than $r_{c}$ ) can the as-formed active phase survives from fluctuation and stays stable. Let us now formulate each component in Eq. (1).

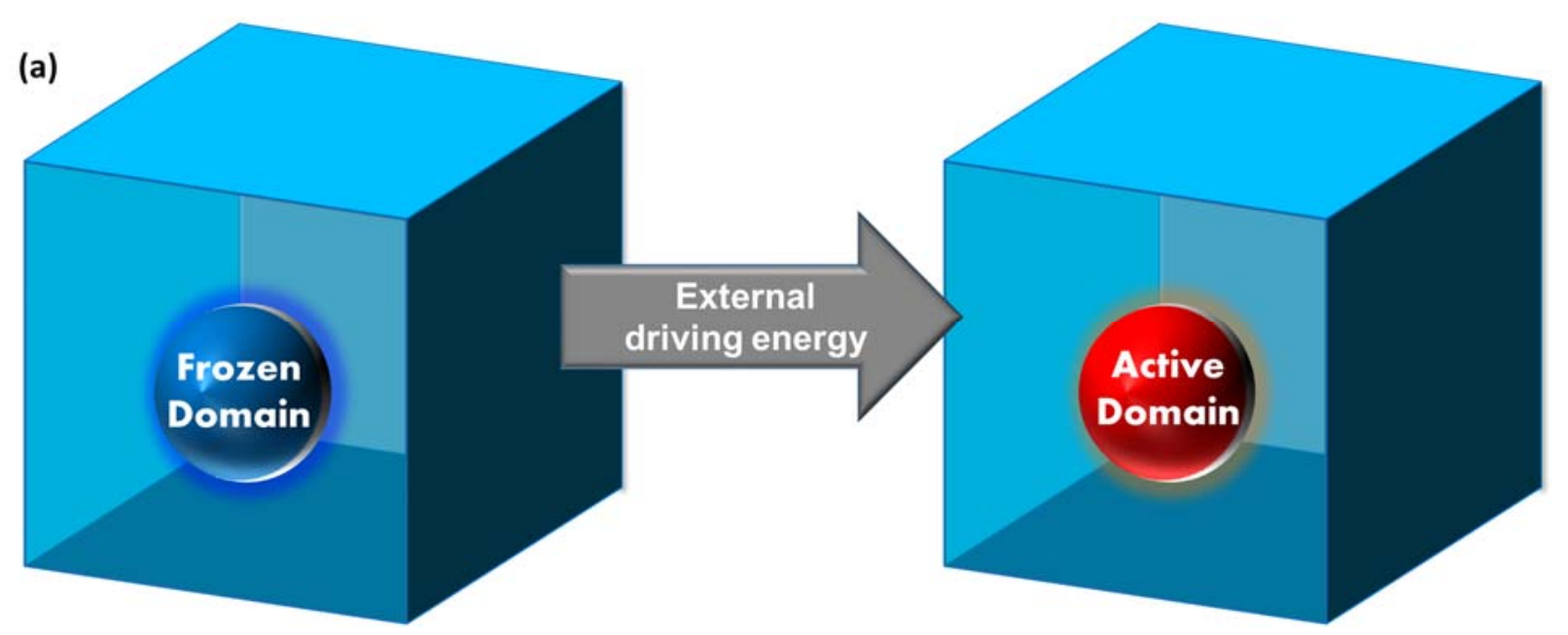


(b)

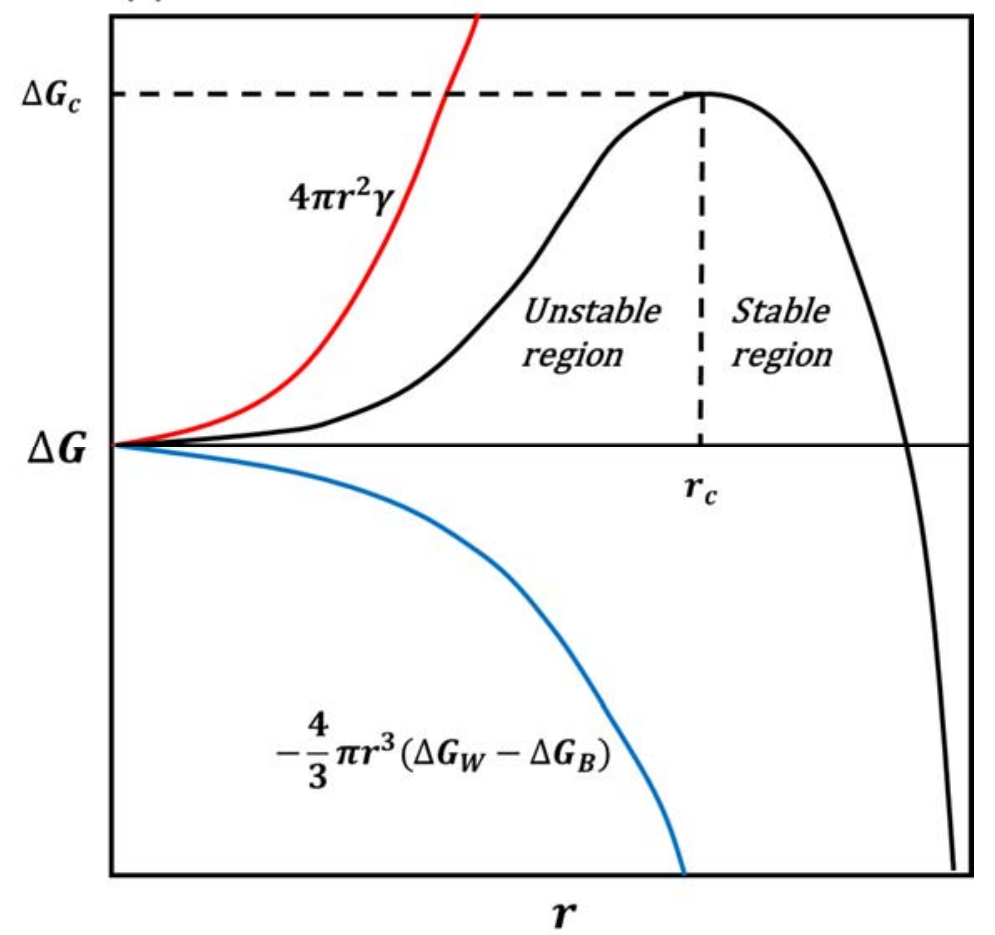

Fig. 1. (a) Schematic illustration of how a frozen domain (blue sphere) in the frozen phase matrix (blue cube) evolves into an active domain (red sphere). (b) The free energy change caused by the appearance of the active domain as a function of domain radius $r . \Delta G_{c}$ is the energy change when the domain size reaches the critical value $r_{c}$. The domains with $r>r_{c}$, will be stabilized in the active phase (and may grow infinitely upon perturbation); while the domains with $r<r_{c}$ will revert back to the frozen phase upon fluctuations.

As we know, the Gibbs free energy change (unit volume) is defined as:

$$
\Delta G_{B}=\Delta H_{B}-T \Delta S_{B}
$$

where $\Delta H_{B}$ is the enthalpy change and $\Delta S_{B}$ is the entropy change. In the frozen phase, the molecular chains can hardly move; they can only vibrate near their equilibrium positions. In the active phase, on the contrary, the molecular chains are able to move cooperatively. To trigger this motion, activation energy with the amount of $\Delta H_{a}$ for each structural unit (i.e. polymer segments) is needed. Studies have shown that the activation energy $\Delta H_{a}$ is temperature dependent (Bauer et al., 2013) and the enthalpy change thus has the simple form:

$$
\Delta H_{B}(T)=n N \Delta H_{a}=n N \frac{A k_{B}}{\left(1-T_{r} / T\right)^{2}}
$$

where $n$ is the molecular chain density (number of molecular chains in unit volume), $N$ is the number of unit segments (Kuhn segments) in one molecular chain and $k_{B}$ is the Boltzmann constant. $A$ and $T_{r}$ are constants and can be determined by linear dielectric spectroscopy (Gainaru et al., 2011; Kudlik et al., 1999; Lunkenheimer et al., 2010).

To detail the entropy contribution to the free energy change, we make the following assumptions: firstly, we neglect the vibrational entropy change and only consider the configurational entropy change; secondly, suppose that, in the frozen state, the 
configurational entropy component can be ignored, in other words, the polymer chains cannot form various configurations; finally, in the active state, the polymer chains act as idea chains and the probability density has the form of Gaussian distribution. We can take the value of the end-to-end distance $L$ to calculate the entropy. Bearing these in mind, the average entropy contribution $\Delta G_{S}$ to the free energy change of $n$ molecular chains with $N$ segments of equal length $l$ is:

$$
\Delta G_{S}=-T \Delta S_{B}=<\frac{3}{2} n k_{B} T \lambda^{2}>\approx \frac{3}{2} n k_{B} T
$$

where the stretch $\lambda=\mathrm{L} / L_{0}$, and $L_{0}=\sqrt{N} l$ is the random-walk root-mean-square distance of a chain. We simply take the average value of $\bar{\lambda}=1$ into Eq. (4) to estimate the average entropy contribution.

In summary, the difference in bulk free energy between a unit frozen domain and an active domain is:

$$
\Delta G_{B}=n k_{B}\left(\frac{N A}{\left(1-T_{r} / T\right)^{2}}+\frac{3}{2} T\right)
$$

As pointed out previously, the source of external work can be heat, force, light and humidity, etc. For the external thermal work density $g_{t w}$, suppose that in the frozen phase, the polymer segments only vibrate more drastically upon heating without change the configuration before the phase transformation, this portion of work is thus considered to be proportional to the temperature, i.e., $g_{t w}=c_{t w} T$. Regarding the external mechanical work $g_{m w}$, it has been observed before that stress can also induce glass transition and this phenomena was explained by the generalized Eyring's ideas of stress-dependent rearrangement activation energies (Eyring, 1936; Patashinski, 2014; Tobolsky and Eyring, 1943; Zhou et al., 1995). Therefore, this term cannot be ignored, especially in cold programming procedure where the pre-stress is high. When consider the non-thermal triggered SMPs, such as photo-activated or water-activated SMPs, work done by light $g_{l w}$ or humidity $g_{h w}$ should be taken into consideration. Then, the change in free energy by the external work takes the form:

$$
\Delta G_{W}=g_{t w}+g_{m w}+g_{l w}+g_{h w}+\cdots
$$

Plug Eq. (5) and (6) back into Eq. (1), we could obtain the expression for the total free energy change $\Delta G$, and hence the equation for the critical size $r_{c}$ in terms of $T$ :

$$
r_{c}=r_{c}(T)=\frac{2 \gamma}{\Delta G_{W}(T)-\Delta G_{B}(T)}
$$

Also, as mentioned before, the whole system consists of domains with different sizes, and we assume their sizes obey quasi-Gaussian distribution, which is commonly used in polymer science (Derrida, 1980; Porter, 1995; Sharafi and Li, 2015; Shojaei and Li, 2014):

$$
p(r)=\left\{\begin{array}{cc}
p_{0} \exp \left(-\frac{(r-\bar{r})^{2}}{2 \Sigma^{2}}\right) & r \geq 0 \\
0 & r<0
\end{array}\right.
$$

where $\bar{r}$ is the average radius, $\Sigma$ is the standard deviation and $p_{0}$ is the normalization factor. The fraction of stable active phase at a certain temperature can thus be expressed as:

$$
f_{1}(T)=\int_{r_{c}(T)}^{\infty} p(r) d r
$$

In addition to the size requirement, the observation time scale $\Delta t=\Delta T / \dot{T}$ should be large enough so that the transition between different energy levels can be finished. More specifically, larger $\Delta t$ indicates slower heating/cooling process, while smaller $\Delta t$ means the heating/cooling rate is higher. According to the transition state theory (Gupta and 
Mauro, 2007; Zwanzig, 2001), the probability $f_{2}$ of the transition from frozen to active phase at temperature $T$ and in observation time $\Delta t$ is:

$$
f_{2}(T, \Delta t)=1-\left[1-\exp \left(-\frac{\Delta H_{a}(T)}{k_{B} T}\right)\right]^{\frac{\Delta t}{\tau_{0}}}
$$

Here, $\tau_{0}$ is the internal time scale for the transition with energy barrier $\Delta H_{a}(T)$.

The two requirements (domain size and observation time) have to be satisfied simultaneously. Therefore, the active phase fraction at a temperature $T$ with heating rate $\dot{T}$ can be expressed as:

$$
\phi_{a}(T, \dot{T})=f_{1} f_{2}=\int_{r_{C}(T)}^{\infty} p(r) d r \times\left\{1-\left[1-\exp \left(-\frac{\Delta H_{a}(T)}{k_{B} T}\right)\right]^{\frac{\Delta t}{\tau_{0}}}\right\}
$$

Accordingly, the frozen phase fraction is:

$$
\left.\phi_{f}(T, \dot{T})=1-\phi_{a}=1-\int_{r_{c}(T)}^{\infty} p(r) d r\right) \times\left\{1-\left[1-\exp \left(-\frac{\Delta H_{a}(T)}{k_{B} T}\right)\right]^{\frac{\Delta t}{\tau_{0}}}\right\}
$$

\section{Constitutive relations}

As stated previously, the whole material is regarded as a two-phase composite whose volume fractions are temperature and rate dependent and obey the evolution law described in the last section. The evolution law plays a central role in the model: the overall elastic behavior, thermal expansion and most importantly, the shape memory effect are highly related to the frozen volume fraction and are detailed in the following three sections.

\subsection{Effective elastic stiffness}

When heating the SMPs, more and more frozen domains evolve into active domains. It is then reasonable to consider the system as a two-phase composite with random distributions of active (rubbery-like) inclusions in a frozen (glassy-like) matrix. Therefore, the Mori-Tanaka approach can be used to predict the effective elastic properties of SMPs. By assuming isotropic spherical inclusion and isotropic matrix, the formulae for the effective properties take the simple form derived by Weng (Weng, 1984):

where,

$$
\begin{gathered}
\bar{K}=K_{f}\left(1+\frac{\phi_{a}\left(K_{a} / K_{f}-1\right)}{1+\alpha_{1} \phi_{f}\left(K_{a} / K_{f}-1\right)}\right) \\
\bar{G}=G_{f}\left(1+\frac{\phi_{a}\left(G_{a} / G_{f}-1\right)}{1+\alpha_{1} \phi_{f}\left(G_{a} / G_{f}-1\right)}\right)
\end{gathered}
$$

$$
\begin{gathered}
\alpha_{1}=\frac{1+v_{f}}{3\left(1-v_{f}\right)} \\
\alpha_{2}=\frac{2\left(4-5 v_{f}\right)}{15\left(1-v_{f}\right)}
\end{gathered}
$$

in which $K_{f}, K_{a}$ and $\bar{K}$ are the bulk modulus of the frozen matrix, rubbery inclusion and composite, respectively. $G_{f}, G_{a}$ and $\bar{G}$ are the elastic shear moduli of the matrix, inclusion, and composite, respectively; and $v_{f}$ is the Poisson's ratio of the frozen matrix.

\subsection{Overall thermal expansion}

The thermal expansion of the isotropic two-phase composite has been derived by Levin (Levin, 1967) and generalized by Rosen and Hashin (Rosen and Hashin, 1970): 


$$
\bar{\alpha}=\phi_{f} \alpha_{f}+\phi_{a} \alpha_{a}+\frac{\frac{1}{\bar{K}}-\left(\phi_{f} / K_{f}+\phi_{a} / K_{a}\right)}{1 / K_{f}-1 / K_{a}}\left(\alpha_{f}-\alpha_{a}\right)
$$

Notice that, unlike the model developed by Liu et al., in which $\bar{\alpha}$ was fitted empirically to experimental results, in the present work, $\bar{\alpha}$ is closely related to both the volume fraction and the elastic properties of the two phases. Therefore, it can be determined directly once the evolution law and the elastic properties for each phase are determined.

\subsection{Shape memory effect}

Shape memory effect also depends largely on the phase evolution law: during the programming or cooling step, the polymer changes from active to frozen phase upon negative temperature increment. In this process, part of the strain that is already reached in the active phase shifts to stress-free strain, namely, storage strain. From a microscopic point of view, the equilibrium molecular configurations received in active state cannot be changed drastically when they transform into frozen state due to the decrease in molecular mobility. To satisfy the boundary condition, stress is then re-distributed throughout the whole system. In the recovery or heating step, the stored strain is released when the frozen phase transforms into an active phase. Depending on the boundary condition, either zero load or zero deformation, shape recovery or stress recovery can be observed. The constitutive relations in the thermomechanical cycle can be formulated based on the above understanding of the shape memory mechanism.

\subsubsection{Programming/Cooling step}

According to the above analysis, during cooling the behavior of the polymer takes the form:

$$
\boldsymbol{\sigma}=\overline{\boldsymbol{L}}:\left(\boldsymbol{\varepsilon}-\boldsymbol{\varepsilon}_{t h}-\boldsymbol{\varepsilon}_{s}\right)
$$

with the effective stiffness tensor $\overline{\boldsymbol{L}}$, total strain $\varepsilon$, thermal strain $\varepsilon_{t h}=\int_{T_{0}}^{T} \bar{\alpha}(T) d T$ and storage strain $\varepsilon_{S}=\int_{T_{0}}^{T} d \varepsilon_{S}$.

The stress increment $d \boldsymbol{\sigma}$ and storage strain development $d \boldsymbol{\varepsilon}_{s}$ is coupled together during the programming step and has been derived by Gilormini and Diani (Gilormini and Diani, 2012):

$$
d \boldsymbol{\sigma}=\overline{\boldsymbol{L}}:\left(d \boldsymbol{\varepsilon}-d \boldsymbol{\varepsilon}_{t h}-d \boldsymbol{\varepsilon}_{s}\right)+\left(\left.\frac{\partial \overline{\boldsymbol{L}}}{\partial \phi_{f}}\right|_{T} \phi_{f}{ }^{\prime}+\left.\frac{\partial \bar{L}}{\partial T}\right|_{\phi_{f}}\right): \overline{\boldsymbol{L}}^{-1}: \boldsymbol{\sigma} d T
$$

and

$$
d \boldsymbol{\varepsilon}_{s}=-\left.\frac{\partial \bar{L}^{-1}}{\partial \phi_{f}}\right|_{T}: \sigma \phi_{f}^{\prime} d T
$$

in which $\phi_{f}{ }^{\prime}$ is the partial derivative of $\phi_{f}$ with respect to temperature.

For the simple case of uniaxial load, the equations become:

$$
\begin{gathered}
d \sigma=\bar{E}\left(d \varepsilon-\bar{\alpha}(T) d T-d \varepsilon_{s}+\left(\left.\frac{1}{9 \bar{K}^{2}} \frac{\partial \bar{K}}{\partial \phi_{f}}\right|_{T}+\left.\frac{1}{3 \bar{G}^{2}} \frac{\partial \bar{G}}{\partial \phi_{f}}\right|_{T}\right) \sigma \phi_{f}{ }^{\prime} d T+\left(\left.\frac{1}{9 \bar{K}^{2}} \frac{\partial \bar{K}}{\partial T}\right|_{\phi_{f}}+\right.\right. \\
\left.\left.\left.\frac{1}{3 \bar{G}^{2}} \frac{\partial \bar{G}}{\partial T}\right|_{\phi_{f}}\right) \sigma d T\right)
\end{gathered}
$$

and 


$$
d \varepsilon_{S}=\left(\left.\frac{1}{9 \bar{K}^{2}} \frac{\partial \bar{K}}{\partial \phi_{f}}\right|_{T}+\left.\frac{1}{3 \bar{G}^{2}} \frac{\partial \bar{G}}{\partial \phi_{f}}\right|_{T}\right) \sigma \phi_{f}{ }^{\prime} d T
$$

During the programming step, the development of the storage strain $d \varepsilon_{s}$ is recorded and will be recalled in the following recovery step.

\subsubsection{Recovery/Heating step}

In the work by Gilormini and Diani (Gilormini and Diani, 2012), the recovery behavior was reproduced by simply 'unrolling the history' of the recorded storage strain. In other words, Eq. (16) can still be applied when $d \boldsymbol{\varepsilon}_{s}$ is not given by Eq. (17), but read from the recorded programming history. Reasonable agreement was obtained using this method. Nevertheless, the real situation is more sophisticated mainly due to the following two reasons:

Firstly, the glass transition, or the phase evolution behavior depends not only on the intrinsic material properties, but also on the experimental conditions, such as, heating rate, applied force, etc. As we have mentioned in Section 2.2.2, the external factors, or the source of input energy, which is used to overcome the energy barrier, can be heat, force, light and humidity, etc. When the boundaries are free (in free recovery tests), only thermal work contributes to the phase transformation process. However, when the boundaries of a test specimen are fixed while the temperature is changing (in programming procedure and fully constraint stress recovery tests), stress and hence mechanical work will be applied to the sample which will serve as part of the input energy source. To this end, in Equation (6), in addition to the thermal energy component $g_{t w}$, the mechanical energy component $g_{m w}$ should also be included when the boundaries of the specimen are fixed. This will lead to different temperature dependence of the frozen phase volume fraction in the free recovery tests, and in the fully constraint recovery test. Therefore, instead of simply using $d \boldsymbol{\varepsilon}_{s}$ recorded in the programming step, the storage strain that is released in each step of temperature increment in the free and fully constraint recovery tests are $-\varepsilon_{s} d \phi_{a}^{t}$ and $-\varepsilon_{s} d \phi_{a}^{t m}$, respectively. Here, we use $\phi_{f}^{t m}\left(\phi_{a}^{t m}\right)$ and $\phi_{f}^{t}\left(\phi_{a}^{t}\right)$ to represent the frozen (active) phase volume fraction functions for the case when both thermal and mechanical work are considered (fixed boundary) and for the case when only thermal work is included (free boundaries), respectively.

The second issue appears in the constraint stress recovery process. Unlike the free strain recovery test, where the recovery strain normally shows a plateau at the end of the test, the stress recovery test is more complicated. In some cases, the recovery stress follows a similar pattern to strain recovery, i.e., gradually approaches to a plateau (Ivens et al., 2011; Yang and Li, 2014a). In some other cases, however, "stress undershoot" (Arrieta et al., 2014; Liu et al., 2006; Tobushi et al., 2001; Véchambre et al., 2011) and "stress overshoot" (Castro et al., 2010; Li and Nettles, 2010; Li and Uppu, 2010; Qi et al., 2008) are observed. Considering the fact that in a two-phase composite SMP, when the composite is subjected to stress, active phase can make self-adjustment instantly, in other words, it behaves elastically; at temperature well below glass transition, the frozen phase does not have enough time to make adjustment, thus also has elastic behavior. However, when temperature is within the transition region, the relaxation time of the frozen phase is comparable to the observation time. Therefore, in addition to thermal expansion and shape 
recovery, stress relaxation must be taken into consideration when modeling the stress recovery process.

It is emphasized here that, as a constitutive equation, it should be independent of the boundary conditions. This is the case for the free shape recovery. However, for constrained shape recovery, the constitutive equation (recovery stress-recovery strain relation) seems depending on the boundary condition of the specimens. The reason is that different boundary conditions add different levels of stress on top of the recovery stress. As a result, the constitutive equation seems depending on the boundary conditions. In other words, in free shape recovery, we are testing "materials" (no boundary conditions are involved); in constrained shape recovery, we are testing "structures" (boundary conditions are involved).

The relaxed stress $\sigma_{\text {relx }}$ normally takes the form of multiple relaxation procedures (Messé et al., 2001; Nguyen et al., 2010; Strobl, 2007; Wang and Li, 2015; Westbrook et al., 2011):

$$
\boldsymbol{\sigma}_{\text {relx }}=\sum_{i=1}^{n} \boldsymbol{\sigma}_{\text {relx }}^{i}=\sum_{i=1}^{n} \boldsymbol{\sigma}_{i}\left(1-\exp \left(-\frac{t}{\tau_{i}}\right)\right)
$$

In our model, the relaxation processes caused by thermal deformation (corresponds to structural relaxation) and mechanical deformation, or releasing of the storage strain (corresponds to stress relaxation) are considered separately, as suggested by preliminary studies (Li and Xu, 2011; Nguyen et al., 2010; Nguyen et al., 2008). Then, we use $n=2$ to represent the relaxation mechanisms caused by thermal and mechanical deformation, respectively. The relaxed stress component corresponds to thermal deformation or structural relaxation is named as $\boldsymbol{\sigma}_{\text {relx }}^{\text {th }}$; similarly, the relaxed stress component related to mechanical deformation or releasing of the storage strain is defined as $\boldsymbol{\sigma}_{\text {relx }}^{\text {rel }}$.

For $\sigma_{\text {relx }}^{\text {th }}$, the simplest model is applied (Westbrook et al., 2011):

$$
\boldsymbol{\sigma}_{\text {relx }}^{\text {th }}=\phi_{f}^{t m} \boldsymbol{\sigma}_{\text {th }}^{\text {eff }}\left(1-\exp \left(-\frac{t}{\tau_{t h}}\right)\right)
$$

here we use an effective stress $\boldsymbol{\sigma}_{t h}^{e f f}$ to represents the stress by thermal expansion only, since it is almost independent of the programming methods; the temperature dependent relaxation time is $\tau_{t h}=\tau_{t h_{0}} b(T)$, with the shift factor $b(T)=\exp \left(-\theta\left(T-T_{g}\right)\right)$.

Regarding $\boldsymbol{\sigma}_{\text {relx }}^{\text {rel }}$, a similar method is applied (Westbrook et al., 2011):

$$
\boldsymbol{\sigma}_{\text {relx }}^{r e l}=\phi_{f}^{t m} \boldsymbol{\sigma}_{\text {rel }}\left(1-\exp \left(-\frac{t}{\tau_{r e l}}\right)\right)
$$

instead of an effective stress, the current releasing stress $\boldsymbol{\sigma}_{\text {rel }}$ (stress caused by releasing of storage strain only, as will be detailed in Table 2) is used, since $\boldsymbol{\sigma}_{r e l}$ depends largely on programming method; the temperature dependent relaxation time is $\tau_{r e l}=\tau_{r e l_{0}} a(T)$ and the shift factor $a(T)$ obeys the Williams-Landel-Ferry (WLF) equation: $\log a(T)=$ $\frac{C_{1}\left(T-T_{g}\right)}{C_{2}+\left(T-T_{g}\right)}$.

\subsection{Summary of the model}

Up to this point, the thermomechanical behavior of the amorphous SMP has been analyzed and formulated from a physical perspective. For completeness, the important features of the model are summarized in Table 2. 
Table 2

Summary of the model

Phase Evolution Law

$\phi_{f}(T, \dot{T})=1-\phi_{a}=1-\left(\int_{r_{c}(T)}^{\infty} p(r) d r\right) \times\left(1-\left(1-\exp \left(-\frac{\Delta H_{a}(T)}{k_{B} T}\right)\right)^{\frac{\Delta t}{\tau_{0}}}\right)$

Energy components

$$
\begin{aligned}
& r_{c}=\frac{2 \gamma}{\Delta G_{W}(T)-\Delta G_{B}(T)} \\
& \Delta G_{B}=n k_{B}\left(\frac{N A}{\left(1-T_{r} / T\right)^{2}}+\frac{3}{2} T\right) \\
& \Delta G_{W}=g_{t w}+g_{m w}+g_{h w}+g_{l w}+\cdots \\
& \Delta H_{a}(T)=\frac{A k_{B}}{\left(1-T_{r} / T\right)^{2}}
\end{aligned}
$$

Thermomechanical properties

Elastic Properties

$$
\begin{aligned}
& \bar{K}=K_{f}\left(1+\frac{\phi_{a}\left(K_{a} / K_{f}-1\right)}{1+\alpha_{1} \phi_{f}\left(K_{a} / K_{f}-1\right)}\right) \\
& \bar{G}=G_{f}\left(1+\frac{\phi_{a}\left(G_{a} / G_{f}-1\right)}{1+\alpha_{1} \phi_{f}\left(G_{a} / G_{f}-1\right)}\right) \\
& \alpha_{1}=\frac{1+v_{\mathrm{f}}}{3\left(1-v_{\mathrm{f}}\right)} \\
& \alpha_{2}=\frac{2\left(4-5 v_{\mathrm{f}}\right)}{15\left(1-v_{\mathrm{f}}\right)}
\end{aligned}
$$

Thermal Expansion

$$
\bar{\alpha}=\phi_{f} \alpha_{f}+\phi_{a} \alpha_{a}+\frac{\frac{1}{\overline{\bar{K}}}-\left(\phi_{f} / K_{f}+\phi_{a} / K_{a}\right)}{1 / K_{f}-1 / K_{a}}\left(\alpha_{f}-\alpha_{a}\right)
$$

Shape Memory effect

Programming/Cooling Step

$$
\begin{aligned}
& d \boldsymbol{\sigma}=\overline{\boldsymbol{L}}:\left(d \boldsymbol{\varepsilon}-d \boldsymbol{\varepsilon}_{t h}-d \boldsymbol{\varepsilon}_{s}\right)+\left(\left.\frac{\partial \overline{\boldsymbol{L}}}{\partial \phi_{f}}\right|_{T} \phi_{f}^{t m^{\prime}}+\left.\frac{\partial \overline{\boldsymbol{L}}}{\partial T}\right|_{\phi_{f}^{t m}}\right): \overline{\boldsymbol{L}}^{-1}: \boldsymbol{\sigma} d T \\
& d \boldsymbol{\varepsilon}_{s}=-\left.\frac{\partial \overline{\boldsymbol{L}}^{-1}}{\partial \phi_{f}}\right|_{T}: \boldsymbol{\sigma} \phi_{f}^{t m^{\prime}} d T
\end{aligned}
$$

Free Recovery Step

$$
\begin{aligned}
& d \boldsymbol{\varepsilon}=d \boldsymbol{\varepsilon}_{t h}+d \boldsymbol{\varepsilon}_{r e l} \\
& d \boldsymbol{\varepsilon}_{r e l}=-\boldsymbol{\varepsilon}_{s} d \phi_{a}^{t} \\
& d \boldsymbol{\varepsilon}_{t h}=\bar{\alpha} d T
\end{aligned}
$$

Constraint Recovery Step

$$
\begin{aligned}
& \boldsymbol{\sigma}=\boldsymbol{\sigma}_{\text {rec }}-\boldsymbol{\sigma}_{\text {relx }} \\
& d \boldsymbol{\sigma}_{\text {rec }}=\overline{\boldsymbol{L}}:\left(d \boldsymbol{\varepsilon}-d \boldsymbol{\varepsilon}_{t h}-d \boldsymbol{\varepsilon}_{r e l}\right)+\left(\left.\frac{\partial \overline{\boldsymbol{L}}}{\partial \phi_{f}}\right|_{T} \phi_{f}{ }^{\prime}+\left.\frac{\partial \overline{\boldsymbol{L}}}{\partial T}\right|_{\phi_{f}}\right): \overline{\boldsymbol{L}}^{-1}: \boldsymbol{\sigma}_{r e c} d T \\
& \boldsymbol{\sigma}_{\text {relx }}^{t h}=\boldsymbol{\sigma}_{e f f}^{t h}\left(1-\exp \left(-\frac{\Delta t}{\tau_{t h}}\right)\right) \phi_{f}^{t m} \\
& \boldsymbol{\sigma}_{\text {relx }}^{\text {rel }}=\boldsymbol{\sigma}_{\text {rel }}\left(1-\exp \left(-\frac{\Delta t}{\tau_{r e l}}\right)\right) \phi_{f}^{t m} \\
& d \boldsymbol{\sigma}_{r e l}=\overline{\boldsymbol{L}}:\left(d \boldsymbol{\varepsilon}-d \boldsymbol{\varepsilon}_{r e l}\right)+\left.\frac{\partial \overline{\boldsymbol{L}}}{\partial \phi_{f}}\right|_{T}: \overline{\boldsymbol{L}}^{-1}: \boldsymbol{\sigma}_{r e c} \phi_{f}{ }^{\prime} d T
\end{aligned}
$$

\section{Results and discussions}

In the work by Liu et al. (Liu et al., 2006), the SMP samples were programmed in uniaxial tension and compression and were then recovered in unconstraint and fully constraint conditions. These experimental results are frequently used for validation of the models established by other researchers (Chen and Lagoudas, 2008a, b; Gilormini and Diani, 2012; Wang et al., 2009), which have exhibit similar prediction abilities compared 
with the model by Liu et al. Here, we also compare our modeling results with the experimental results carried out by Liu et al. (Liu et al., 2006).

\subsection{Parameter determination}

The phase evolution law and the constitutive relations were coded and implemented into the Mathematica program. The flowchart is illustrated in Appendix A to obtain the optimized values for each parameter. Detailed parameter identification procedures are briefly described in Appendix B. The final values of these parameters are listed in Table 3.

Table 3

Parameters used for the present model

\begin{tabular}{|c|c|c|}
\hline Description & Parameters & Values \\
\hline Frozen phase volume fraction & & \\
\hline Average size of the domains & $\bar{r}(n m)$ & 2.5 \\
\hline $\begin{array}{l}\text { Size deviation of the domains } \\
\text { when both external thermal and } \\
\text { mechanical work are considered }\end{array}$ & $\Sigma_{t m}(n m)$ & 0.08 \\
\hline $\begin{array}{l}\text { Size deviation of the domains } \\
\text { when only external thermal } \\
\text { work is considered }\end{array}$ & $\Sigma_{t}(n m)$ & 0.05 \\
\hline Surface tension & $\gamma(N / m)$ & 0.05 \\
\hline $\begin{array}{l}\text { Constant related to external } \\
\text { thermal work density }\end{array}$ & $c_{t m}\left(J /\left(m m^{3} K\right)\right)$ & $1.2 \times 10^{-4}$ \\
\hline $\begin{array}{l}\text { External mechanical work } \\
\text { density }\end{array}$ & $g_{m w}\left(J / m m^{3}\right)$ & $3.6 \times 10^{-3}$ \\
\hline Cross-link density & $n\left(m^{-3}\right)$ & $1.5 \times 10^{25}$ \\
\hline $\begin{array}{l}\text { Number of Kuhn segments } \\
\text { between two crosslinks }\end{array}$ & $N$ & 250 \\
\hline $\begin{array}{l}\text { Constant related to the linear } \\
\text { dielectric spectroscopy of the } \\
\text { material }\end{array}$ & $A$ & 4 \\
\hline $\begin{array}{l}\text { Reference temperature related to } \\
\text { the linear dielectric } \\
\text { spectroscopy of the material }\end{array}$ & $T_{r}(K)$ & 200 \\
\hline Heating rate term & $\Delta t / \tau_{0}$ & 1 \\
\hline $\begin{array}{l}\text { Thermomechanical properties } \\
\text { Young's modulus for active } \\
\text { phase }\end{array}$ & $E_{a}(M P a)$ & $(0.025 T)$ \\
\hline $\begin{array}{l}\text { Young's modulus for frozen } \\
\text { phase }\end{array}$ & $E_{f}(M P a)$ & 750 \\
\hline Poison's ratio for active phase & $v_{a}$ & 0.498 \\
\hline Poison's ratio for frozen phase & $v_{f}$ & 0.4 \\
\hline $\begin{array}{l}\text { Coefficient of thermal } \\
\text { expansion for active phase }\end{array}$ & $\alpha_{a}\left(K^{-1}\right)$ & $1.8 \times 10^{-4}$ \\
\hline $\begin{array}{l}\text { Coefficient of thermal } \\
\text { expansion for frozen phase }\end{array}$ & $\alpha_{f}\left(K^{-1}\right)$ & $0.9 \times 10^{-4}$ \\
\hline Glass transition & $T_{g}(K)$ & 343 \\
\hline $\begin{array}{l}\text { Stress relaxation components } \\
\text { Relaxation time for thermal } \\
\text { expansion }\end{array}$ & $\tau_{t h_{0}}(s)$ & 0.1 \\
\hline $\begin{array}{l}\text { Material temperature parameter } \\
\text { for thermal expansion }\end{array}$ & $\theta\left(K^{-1}\right)$ & 0.1 \\
\hline $\begin{array}{l}\text { Relaxation time for shape } \\
\text { recovery }\end{array}$ & $\tau_{r e l_{0}}(s)$ & 1 \\
\hline $\begin{array}{l}\text { WLF constant } \\
\text { WLT constant }\end{array}$ & $\begin{array}{l}C_{1} \\
C_{2}(K)\end{array}$ & $\begin{array}{l}15 \\
350\end{array}$ \\
\hline
\end{tabular}




\subsection{Comparison with experimental results}

To have a general idea on the evolution law discussed above, we first illustrate in Fig. 2 the frozen phase volume fraction functions $\phi_{f}$ in both cases with the external mechanical work $\phi_{f}^{t m}$ and without the external mechanical work $\phi_{f}^{t}$, together with the results obtained by Liu et al. (Liu et al., 2006) as a comparison. Notice that the temperature has been normalized by the glass transition temperature (343K) tested by Liu et al. (2006). As can be seen from Fig. 2, since both Liu's result and $\phi_{f}^{t}$ in this study were obtained from the free recovery test, they have very similar behavior. With the external mechanical work, the transition starts earlier and is smoother. Although Liu et al.'s phase evolution law possesses a simpler form, it is unable to account for the different conditions due to the phenomenological nature of their model. Our phase evolution law, on the contrary, instead of fitting directly onto the experimental results, starts from the fundamental physical law, uses the intrinsic parameters of the material, considers the influence of different situations, and can accurately reproduce the frozen phase volume fraction in various cases.

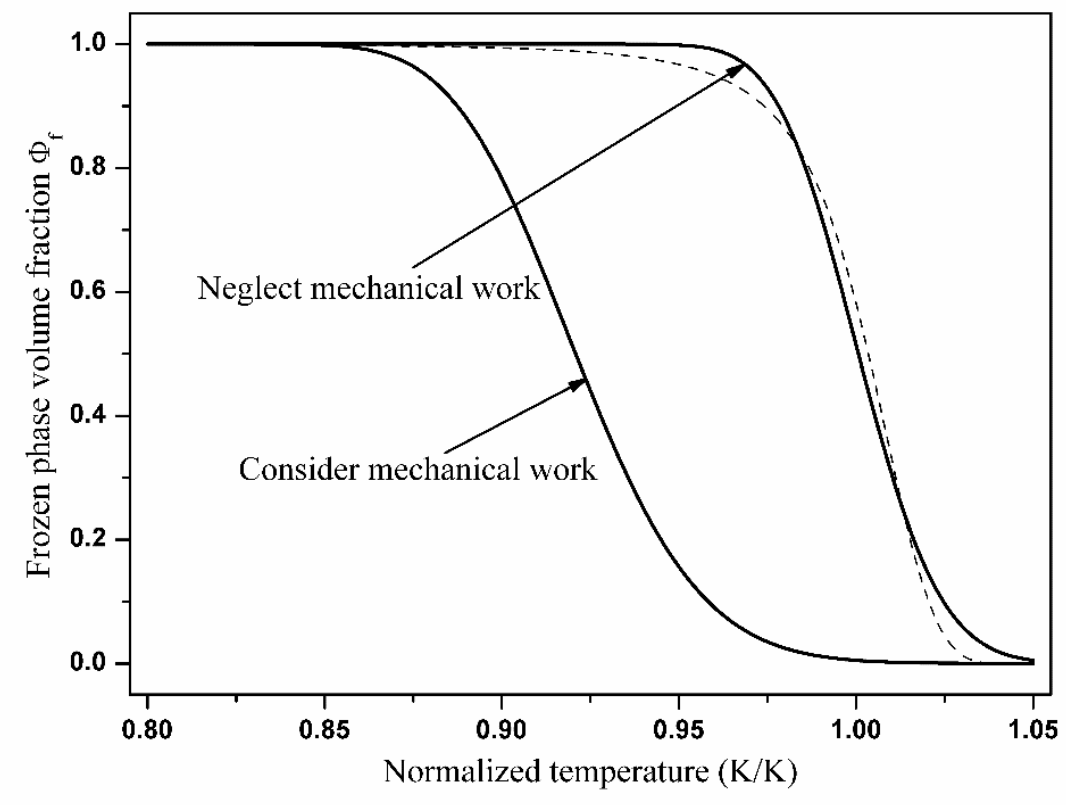

Fig. 2. Frozen phase volume fraction in both cases with and without the external mechanical work (solid lines), together with the modeling result by Liu et al. (Liu et al., 2006) (dashed line).

Using the frozen phase volume fraction which considered the mechanical work $\phi_{f}^{t m}$, the stress response during cooling can be reproduced. Fig. 3 shows modeling results of the stress responses of the SMPs under different pre-strain conditions. The experiment carried out by Liu et al. and their modeling results are also provided as a comparison. Clearly, our model shows a better agreement with the experimental results as compared to their model. We believe that the more obvious deviation of the model by Liu et al. from the experimental results is mainly due to the fact that the mechanical work was not considered in their model. 


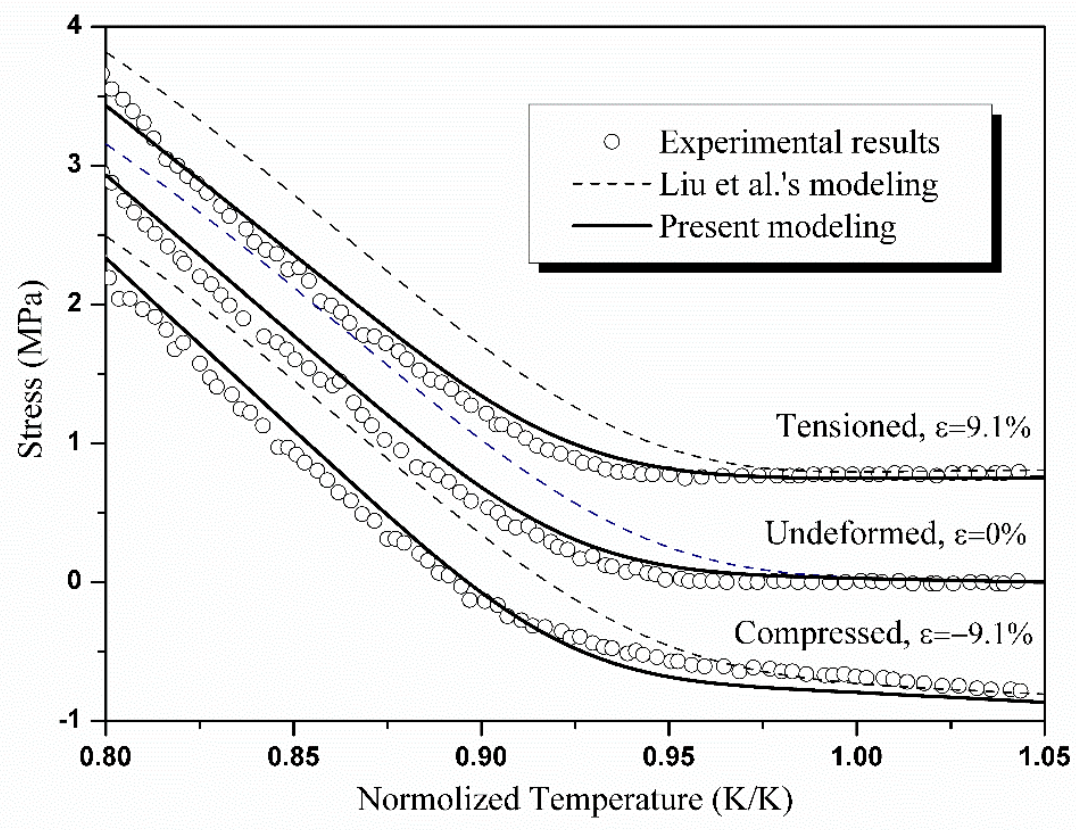

Fig. 3. Modeling results for the stress responses of SMPs during cooling under different prestrain conditions.

It is noted that a slightly stiffer behavior at the lower temperatures can be observed for our model as compared to the test results. The reason for this phenomenon is likely attributed the stiff response of the Mori-Tanaka model used to predict the elastic properties of the polymer (Chaboche et al., 2005; Shojaei and Li, 2013). At lower temperatures, the frozen phase and active phase are stiffer, leading to even stiffer response by the model. Overestimation of the stiffness leads to consistent overestimation of stress at lower temperatures.

Fig. 4 shows the modeling results for the free strain recovery tests of SMPs programmed by different pre-strains. Both our model and Liu's model exhibit good agreement with the experimental results. However, a slightly stiffer behavior at the beginning of the transformation can be observed for our model. One of the many reasons behind this phenomenon may be attributed to our choice of spatial distribution of the frozen domains, i.e., quasi-Gaussian distribution. Integration of this quasi-Gaussian distribution (Equation (8)) then leads to the error function which determines mostly the behavior of the phase evolution law. Development of a more accurate, non-Gaussian spatial distribution model should be conducted in the future for a better description. 


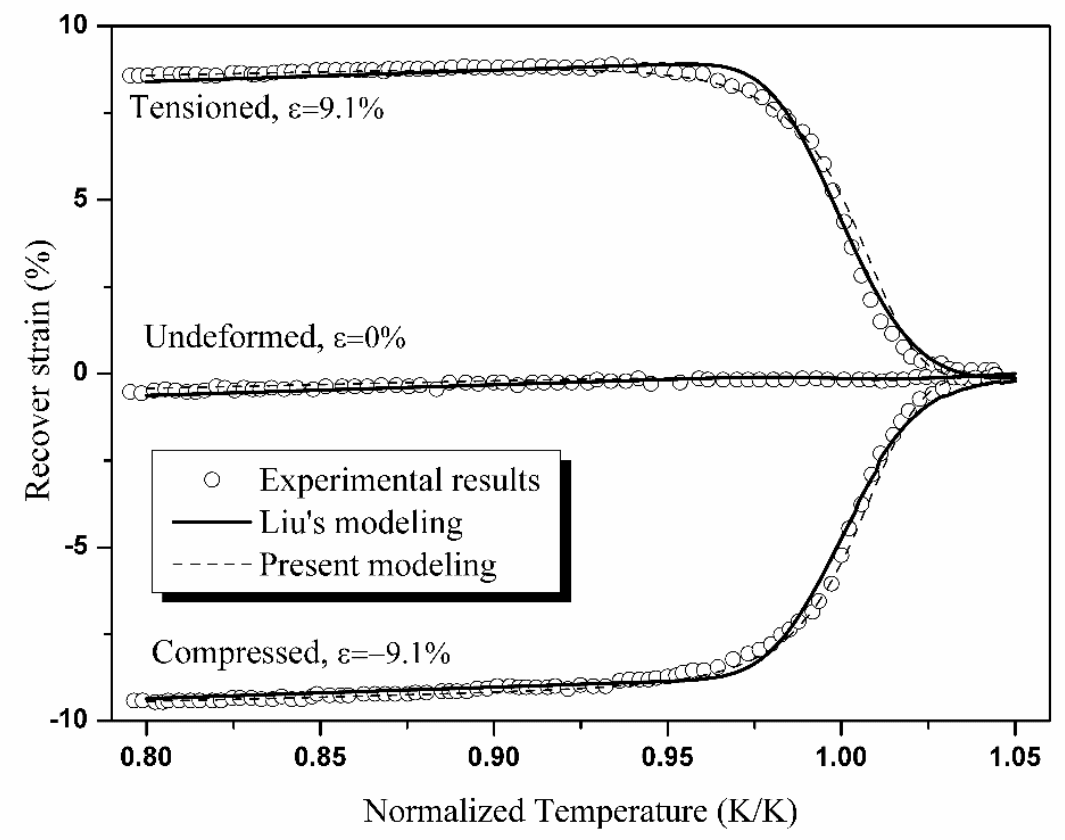

Fig. 4. Modeling results for the free strain recovery tests of SMPs programmed by different pre-strains.

For the fully constraint stress recovery tests, the experimental and modeling results are presented in Fig. 5. Both the present and Liu's models show the ability to reproduce the trend for the stress response in fully constrained condition. Notice that, by taking the stress relaxation into consideration in our model, the stress response during fully constraint recovery of the compression sample can be reproduced more accurately. Nevertheless, the relaxation mechanism may be affected by the thermomechanical history. Therefore, when we use the relaxation parameters obtained from the compression programmed sample to predict the stress recovery behaviors of the un-programmed and tension programmed samples, a certain deviation is observed (See Fig.5). 


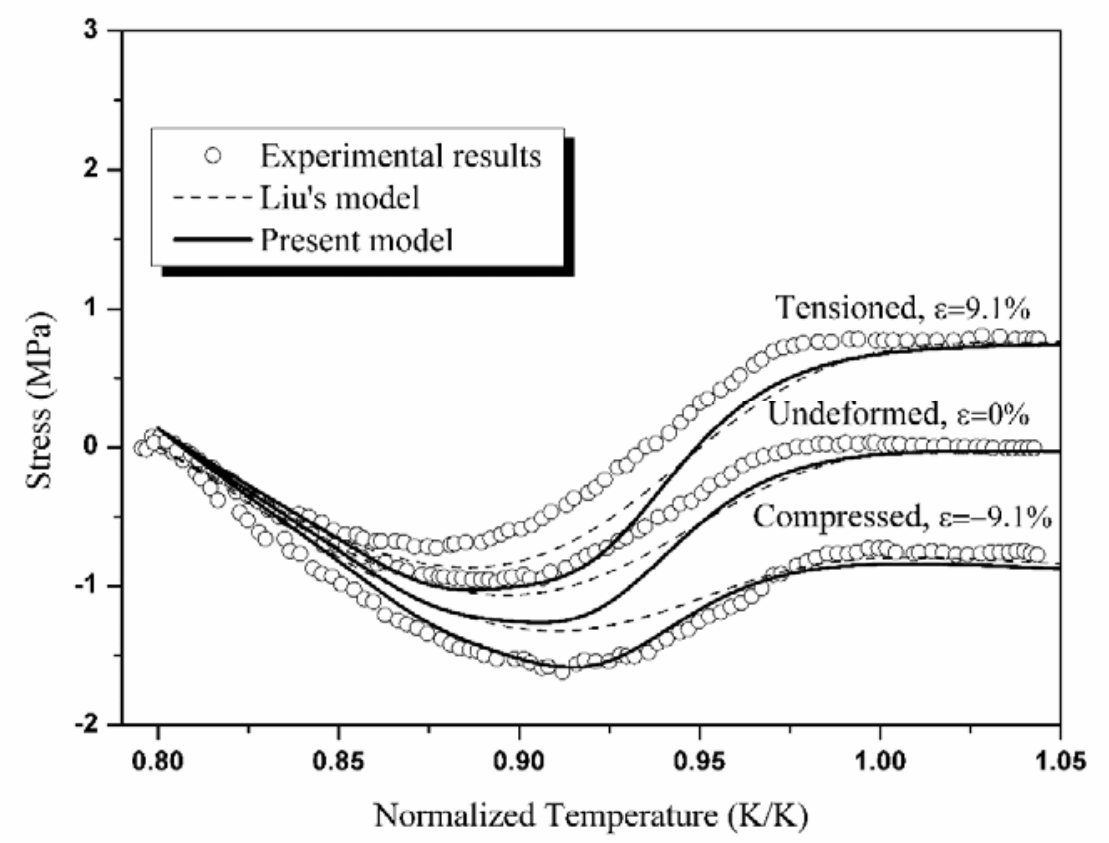

Fig. 5. Modeling results for the constraint stress recovery tests of SMPs programmed by different pre-strains.

\subsection{Parametric study}

As has been proved before (Adam and Gibbs, 1965; Thirtha et al., 2006), the glass transition behavior of amorphous polymers depends largely on their morphologies. In Fig. 6(a) and (b), the influence of the average domain size $\bar{r}$ and standard deviation of the domain size $\Sigma$ defined in Eq. (8) are presented. The results in Fig. 6(a) indicate that the larger the average domain size is, the earlier the transition starts. This is because when the total dynamic heterogeneous domain volume is constant, the system that consists of smaller domains has higher surface energy that need to be overcome. Therefore, smaller sized domains need higher temperature to trigger the transition.

Fig. 6(b) shows that the larger standard deviation of the dynamic heterogeneous domain sizes leads to smoother transition. This is because the larger standard deviation suggests wider distribution of the domain sizes, from very small to very large. Consequently, the transition starts at lower temperature (for larger domain size) and ends at higher temperature (for smaller domain size). In other words, it leads to wider or smoother transition. Hence, a suitable morphology of the polymer is a prerequisite for the proper glass transition behavior.

Another important aspect that affects the phase evolution is the external mechanical work. Although a more accurate method is needed to quantify this portion of work, its necessity has been demonstrated in the above section. As a matter of fact, the stress induced glass transition phenomena has been observed before (Patashinski, 2014; Zhou et al., 1995) which provides a solid foundation for our model. As can be seen from Fig. 6(c), when the external mechanical work is large enough, the frozen-active phase transition can be 
triggered at a temperature well below the glass transition temperature. Therefore, by taking the external mechanical work into consideration, the effect of cold programming (program the sample at a temperature lower than the glass transition zone) on shape memory can also be explained from the point of view of the phase transition. In other words, owing to the large mechanical work during cold-programming ( $\mathrm{Li}$ and $\mathrm{Xu}, 2011$ ), the glass transition temperature has been significantly reduced. Hence, the actual programming temperature is not as cold as it seems.

As has been pointed out in Liu et al.'s work, the heating rate is also a significant factor that determines the phase evolution behavior. In Liu's work, the observation time is comparable with the internal transition time; as a result, the heating was not reflected in their case. However, as shown in Fig. 6(d), if the heating rate is too high, the phase transition may not have enough time to complete. In other words, the shape memory effect cannot be fully displayed in the short observation time.

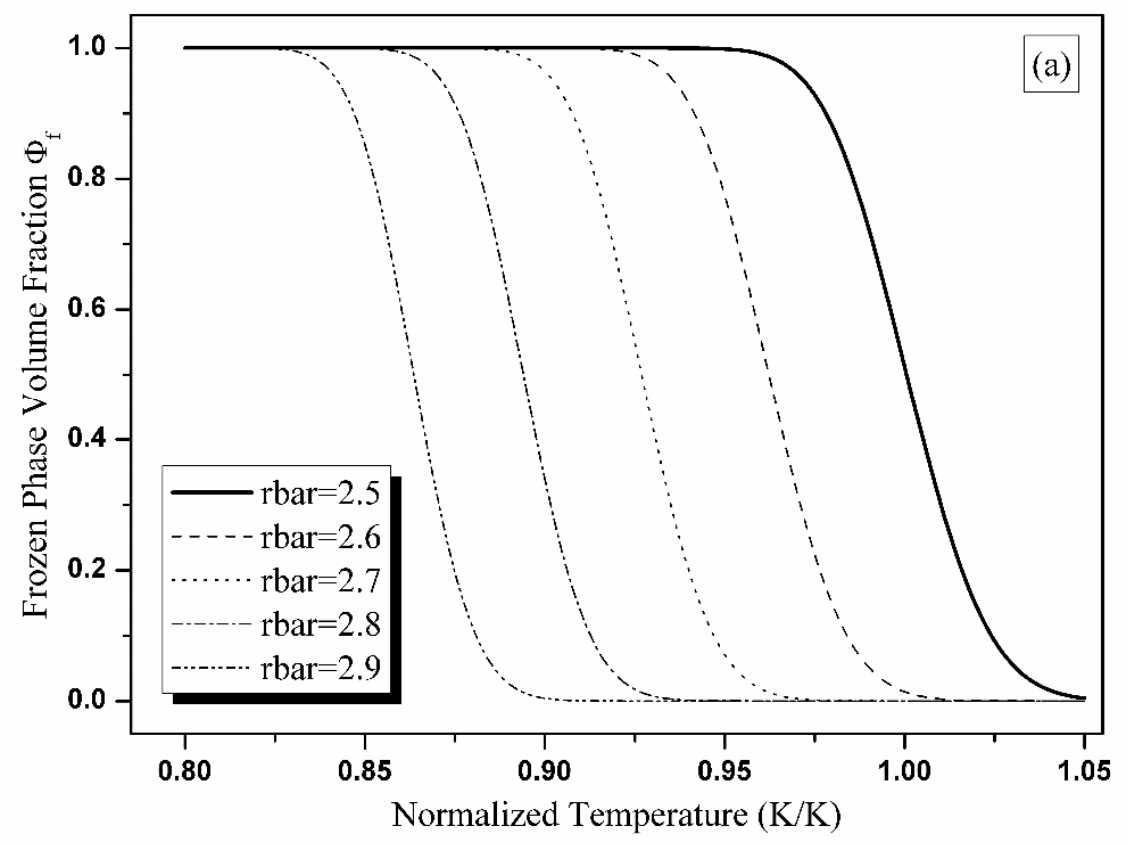



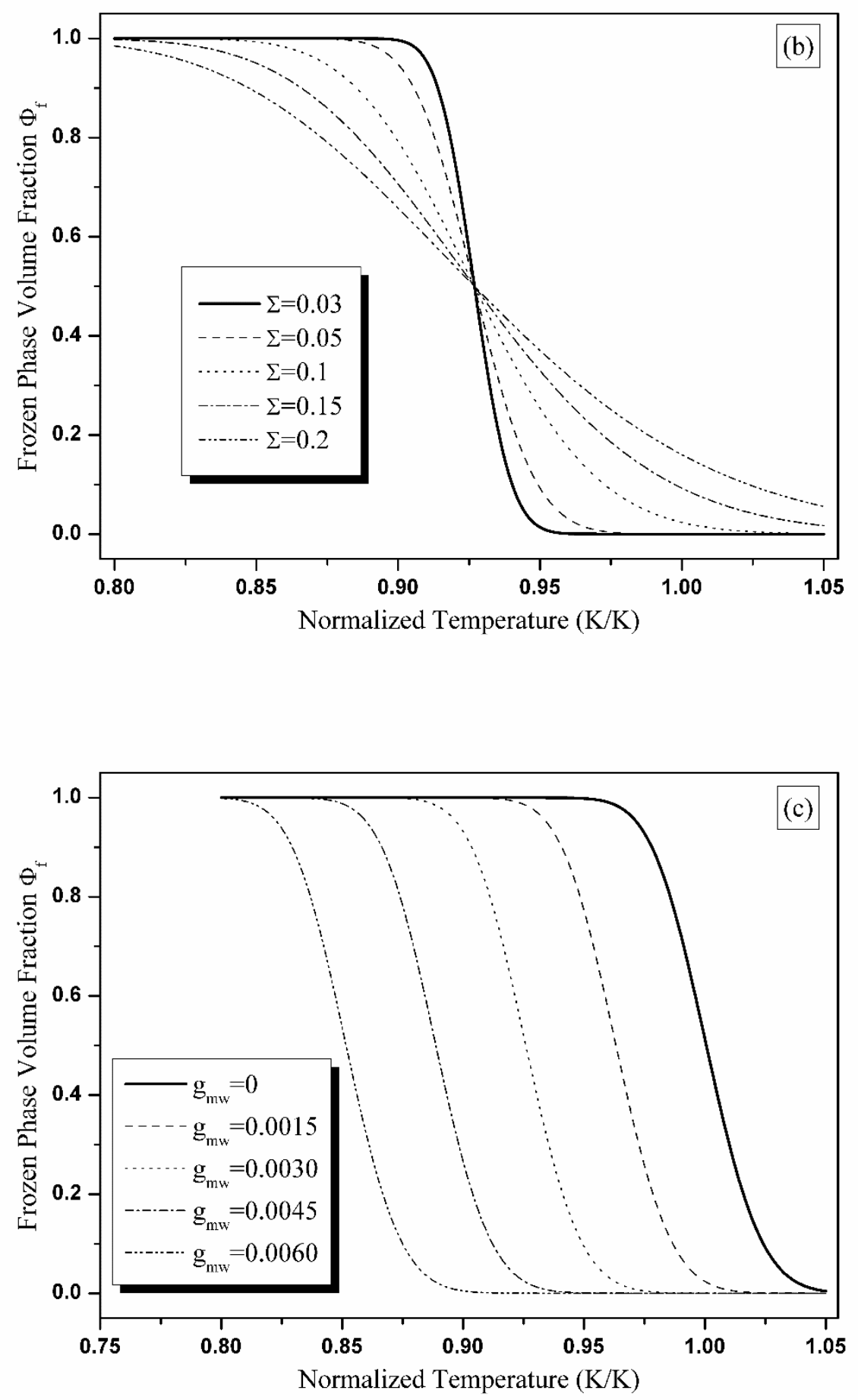


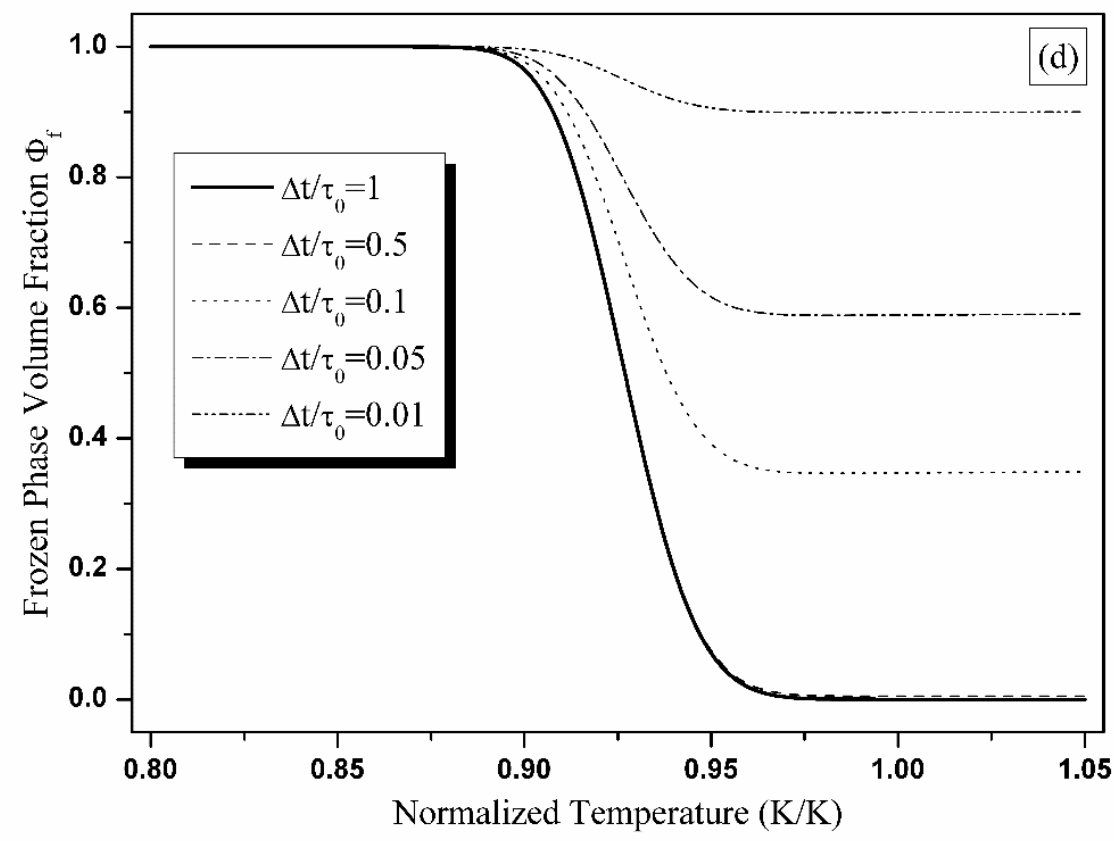

Fig. 6. Prediction of frozen phase fraction with (a) average domain size $\bar{r}$; (b) standard deviation of the domain size $\Sigma$; (c) external mechanical work density $g_{m w}$; and (d) heating rate $\Delta t / \tau_{0}$.

\section{Conclusions}

The phase transition model is one of the most widely used constitutive models for designing amorphous SMPs due to its ease of application. However, a major concern is the lack of physical basis for the curve-fitted phase evaluation law. In this paper, a new physics based phase evaluation law is formulated. The formulation is based on the concept that amorphous SMPs are a two-phase composite material. The free energy change during the programming and recovery governs the shape fixity and shape recovery. The different boundary conditions during free shape recovery and constrained stress recovery are modeled separately. The modeling results are compared with the test and modeling results found in the literature. Parametric studies are also conducted based on the validated model. The following conclusions are obtained:

(1) The model reasonably captured the shape fixity and shape recovery process, governed by clear physics.

(2) It is found that the dynamic heterogeneous domain size distribution has a significant effect on the memory effect.

(3) External mechanical work, which is not considered in previous models, also has a significant effect on the phase evolution, hence, the shape memory behavior. It also provides new insight into the cold programming principles.

(4) The shape recovery depends on the heating rate. The observation time must be sufficient in order to fully demonstrate the shape memory capability. 
While this work focused on amorphous SMPs, the principle and procedure can be easily used in modeling semi-crystalline SMPs, and even polymeric artificial muscles with similar phase transitions.

\section{Acknowledgment}

This study was financially supported by National Science Foundation under grant number CMMI 1333997, the Cooperative Agreement NNX11AM17A between NASA and the Louisiana Board of Regents under contract NASA/LEQSF(2011-14)-Phase3-05, and Army Research Office under grant number W911NF-13-1-0145.

\section{Appendix A}

Our numerical simulation contains two parts: First, we used the "FindFit" function in Mathematica to identify the optimized values for each parameter. Second, our model, together with Liu's model were coded and implemented into Matlab program to calculate and plot the simulation results.

\section{A.1. Programming/Cooling step}

In this step, the experimental results of the stress response during cooling with a prestrain of $\varepsilon_{\text {pre }}=-9.1 \%$ were used to optimize the parameters in $\phi_{f}^{t m}$, which corresponds to frozen phase volume fraction with both external mechanical and thermal work $\left(\Delta G_{W}=\right.$ $\left.g_{t w}+g_{m w}\right)$. The flowchart of the curve fitting procedures is outlined below:

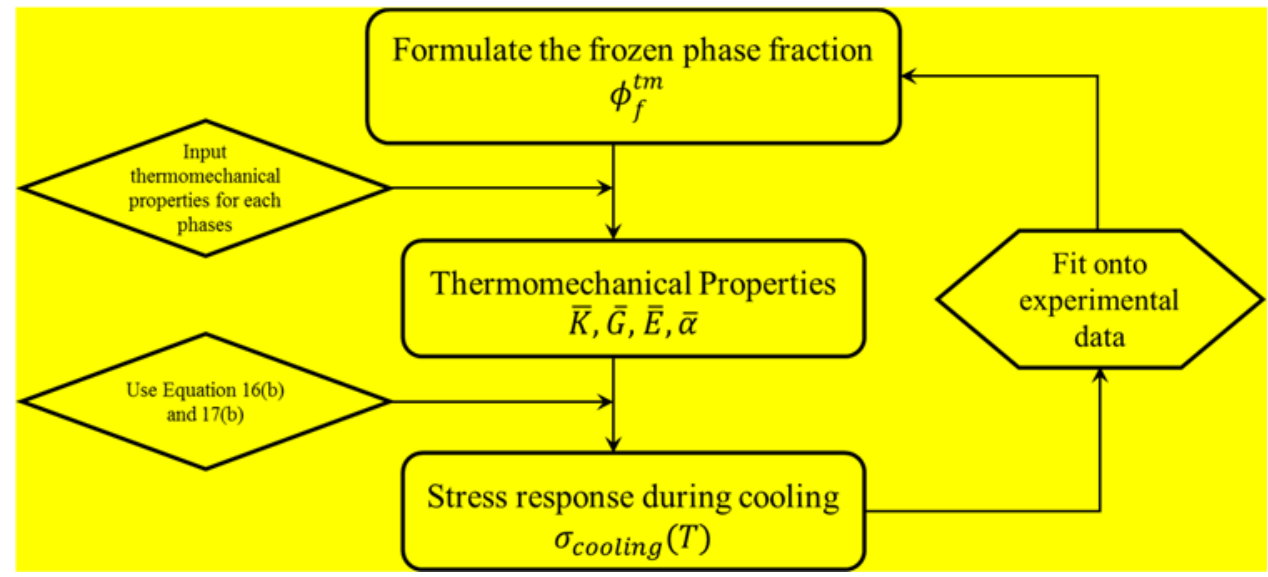

Figure A1. Flowchart of the curve fitting process for $\phi_{f}^{t m}$ using the Mathematica program.

In the next simulation part, this $\phi_{f}^{t m}$ was used as the input frozen phase volume fraction for all the programming and fully constraint recovery tests. Also, notice that, in the programming procedure, the storage strain at each temperature increment $\varepsilon_{S}(T)$ was recorded for the future use. 


\section{A2. Free recovery step}

In this step, the experimental data of the free strain recovery test with a pre-strain of $\varepsilon_{\text {pre }}=-9.1 \%$ was used to obtain $\phi_{f}^{t}$, which corresponds to the frozen phase volume fraction with only external thermal work $\Delta G_{W}=g_{t w}$. The curve fitting process is outlined as follows:

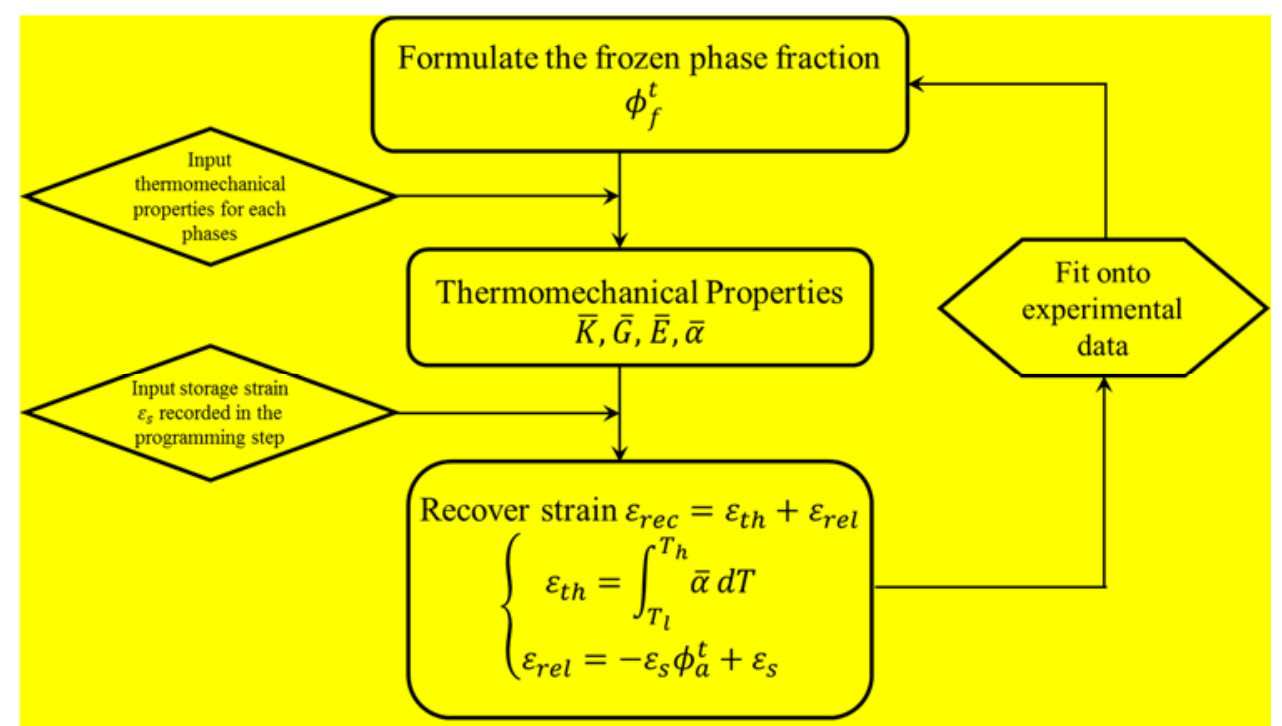

Figure A2. Flowchart of the curve fitting process for $\phi_{f}^{t}$ using the Mathematica program.

In the following simulation part, the optimized $\phi_{f}^{t}$ was used to reproduce all the free strain recovery tests.

\section{A3. Fully constraint stress recovery step}

In this step, the parameters related to the stress relaxation were determined from the stress recovery test result of the $\varepsilon_{\text {pre }}=-9.1 \%$ pre-strain specimen and the curve fitting process is illustrated below: 


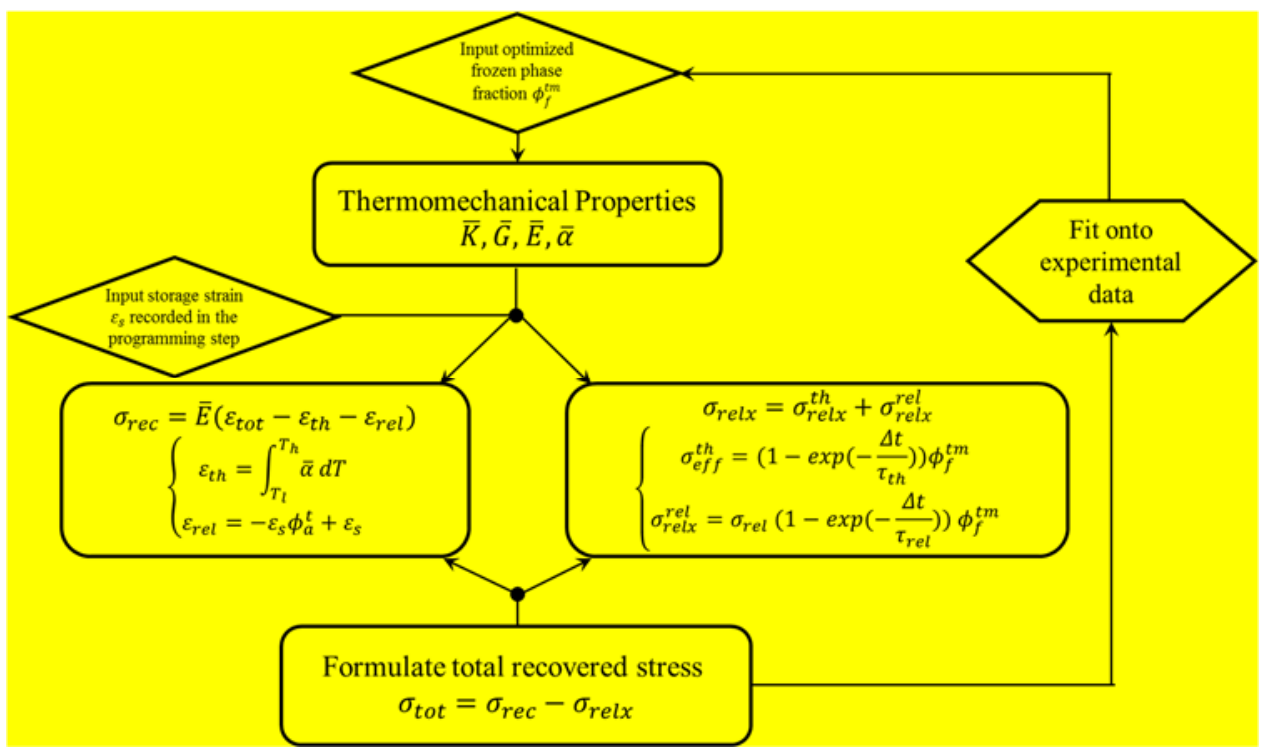

Figure A3. Flowchart of the curve fitting process for the parameters related to the stress relaxation using the Mathematica program.

The optimized parameters related to the stress relaxation $\left(\tau_{t h_{0}}, \theta, \tau_{r e l_{0}}, C_{1}, C_{2}\right)$ were used to reproduce other stress recovery test.

\section{Appendix B}

The test specimens were a commercial thermoset epoxy system, DP5.1 supplied by Composite Technology Development (CTD), Inc. Some material parameters can be determined directly from the standard thermomechanical measurements in (Liu et al., 2006): the coefficient of thermal expansion for the pure active and frozen phase is respectively $\alpha_{a}=1.8 \times 10^{-4} / K$ and $\alpha_{g}=0.9 \times 10^{-4} / K$; the Young's modulus is $E_{a}=$ (0.025T) $M P a$ for non-programmed active phase and $E_{f}=750 M P a$ for non-programmed frozen phase. As has been observed before (Nettles, 2009; Yang and Li, 2014b), the programming step can change the elastic properties to some extents due to the volume change. We found that variation values of $\pm 7 \%$ in Young's modulus for compression programmed and tension programmed samples are reasonable. In the present work, both bulk and shear moduli are required. As suggested by Gilormini and Diani (Gilormini and Diani, 2012), $v_{a}=0.498$ and $v_{f}=0.4$ are appropriate values for Poisson's ratio of the active and frozen phases, respectively. The parameters related to the stress relaxation were determined from the stress recovery test result of the $\varepsilon_{p r e}=-9.1 \%$ pre-strain specimen (Liu et al., 2006).

The procedure to determine the parameters in the frozen phase volume fraction function is more complicated, since these parameters are all related to the intrinsic material properties. However, we have rather limited knowledge about the Epoxy system used in Liu's work. In order to prove the prediction ability of our model and retain the physical meaning of each parameter, we followed two steps to determine the parameter values: first, 
set up a rough initial guess based on our knowledge for similar materials, and second, fit the model onto the experimental results by Liu et al. Both the initial guess and the allowable variation range are within the test results for similar materials in the existing literature. For example, the surface energy for thermoset epoxy is approximately $\gamma=$ $0.05 \mathrm{~N} / \mathrm{m}$ (Comyn, 2006; $\mathrm{Wu}, 1989$ ); the molecular chain density $n$ is roughly equal to the cross-link density (number of cross-link points per unit volume) with a value of $5.9 \times$ $10^{26} \mathrm{~m}^{-3}$ used in Liu et al.'s model (Liu et al., 2006); the reference temperature $T_{r}$ was taken as $200 \mathrm{~K}$, which is an average of the theoretical and experimental values for similar materials in the literature (Paluch, 2000; Schönhals, 1998); according to the recent experimental results (Ediger, 2000; Reinsberg et al., 2001; Shell et al., 2005), the average size of the dynamic heterogeneous domains is of the order of several nanometers and the initial value is taken to be $2 \mathrm{~nm}$; the product of Kuhn segments $N$ and constant $A$ is estimated by the activation energy associated with the glass transition for the Epoxy system and is set as $1000 K$ ( $\mathrm{Li}$ et al., 2000; Vyazovkin et al., 2006). The volume fraction $\phi_{f}^{t}$ was fitted onto the strain recovery test result with a pre-strain of $\varepsilon_{\text {pre }}=-9.1 \%$; and the volume fraction $\phi_{f}^{t m}$ with both external mechanical and thermal work was fitted onto the stress response curve during cooling with a pre-strain of $\varepsilon_{\text {pre }}=-9.1 \%$.

\section{References}

Adam, G., Gibbs, J.H., 1965. On the temperature dependence of cooperative relaxation properties in glass - forming liquids. The Journal of Chemical Physics 43, 139-146.

Andersen, H.C., 2005. Molecular dynamics studies of heterogeneous dynamics and dynamic crossover in supercooled atomic liquids. Proceedings of the National Academy of Sciences of the United States of America 102, 6686-6691.

Arrieta, J.S., Diani, J., Gilormini, P., 2014. Cyclic and monotonic testing of free and constrained recovery properties of a chemically crosslinked acrylate. Journal of Applied Polymer Science $131,39813$.

Auricchio, F., Reali, A., Stefanelli, U., 2007. A three-dimensional model describing stress-induced solid phase transformation with permanent inelasticity. International Journal of Plasticity 23, 207-226.

Baghani, M., Naghdabadi, R., Arghavani, J., Sohrabpour, S., 2012. A thermodynamicallyconsistent 3D constitutive model for shape memory polymers. International Journal of Plasticity 35, 13-30.

Balogun, O., Mo, C., 2014. Shape memory polymers: three-dimensional isotropic modeling. Smart Materials and Structures 23, 045008.

Bauer, T., Lunkenheimer, P., Loidl, A., 2013. Cooperativity and the freezing of molecular motion at the glass transition. Physical Review Letters 111, 225702.

Behl, M., Lendlein, A., 2007. Shape-memory polymers. Materials Today 10, 20-28.

Buckley, C., Prisacariu, C., Caraculacu, A., 2007. Novel triol-crosslinked polyurethanes and their thermorheological characterization as shape-memory materials. Polymer 48, 1388-1396.

Buckley, P.R., McKinley, G.H., Wilson, T.S., Small, W., Benett, W.J., Bearinger, J.P., McElfresh, M.W., Maitland, D.J., 2006. Inductively heated shape memory polymer for the magnetic actuation of medical devices. Biomedical Engineering, IEEE Transactions on 53, 2075-2083.

Castro, F., Westbrook, K.K., Long, K.N., Shandas, R., Qi, H.J., 2010. Effects of thermal rates on the thermomechanical behaviors of amorphous shape memory polymers. Mechanics of TimeDependent Materials 14, 219-241. 
Chaboche, J., Kanouté, P., Roos, A., 2005. On the capabilities of mean-field approaches for the description of plasticity in metal matrix composites. International Journal of Plasticity 21, 1409-1434.

Chae Jung, Y., Hwa So, H., Whan Cho, J., 2006. Water - Responsive Shape Memory Polyurethane Block Copolymer Modified with Polyhedral Oligomeric Silsesquioxane. Journal of Macromolecular Science, Part B 45, 453-461.

Chen, X., Nguyen, T.D., 2011. Influence of thermoviscoelastic properties and loading conditions on the recovery performance of shape memory polymers. Mechanics of Materials 43, 127-138.

Chen, Y.-C., Lagoudas, D.C., 2008a. A constitutive theory for shape memory polymers. Part I: Large deformations. Journal of the Mechanics and Physics of Solids 56, 1752-1765.

Chen, Y.-C., Lagoudas, D.C., 2008b. A constitutive theory for shape memory polymers. Part II: a linearized model for small deformations. Journal of the Mechanics and Physics of Solids 56, 1766-1778.

Chung, T., Romo-Uribe, A., Mather, P.T., 2008. Two-way reversible shape memory in a semicrystalline network. Macromolecules 41, 184-192.

Comyn, J., 2006. in: Cognard, P. (Ed.), Handbook of Adhesives and Sealants. Elsevier, Amsterdam, The Netherlands, p. 34.

Dachkovski, S., Böhm, M., 2004. Finite thermoplasticity with phase changes based on isomorphisms. International Journal of Plasticity 20, 323-334.

Debenedetti, P.G., 1996. Metastable liquids: concepts and principles. Princeton University Press, Princeton, New Jersey.

Derrida, B., 1980. Random-energy model: Limit of a family of disordered models. Physical Review Letters 45, 79.

Diani, J., Gall, K., 2007. Molecular dynamics simulations of the shape-memory behaviour of polyisoprene. Smart Materials and Structures 16, 1575.

Diani, J., Gilormini, P., Frédy, C., Rousseau, I., 2012. Predicting thermal shape memory of crosslinked polymer networks from linear viscoelasticity. International Journal of Solids and Structures 49, 793-799.

Diani, J., Liu, Y., Gall, K., 2006. Finite strain 3D thermoviscoelastic constitutive model for shape memory polymers. Polymer Engineering \& Science 46, 486-492.

Dietsch, B., Tong, T., 2007. A review-: Features and benefits of shape memory polymers (SMPs). Journal of Advanced Materials 39, 3-12.

Ediger, M.D., 2000. Spatially heterogeneous dynamics in supercooled liquids. Annual Review of Physical Chemistry 51, 99-128.

Eyring, H., 1936. Viscosity, plasticity, and diffusion as examples of absolute reaction rates. The Journal of Chemical Physics 4, 283-291.

Fulcher, G.S., 1925. Analysis of recent measurements of the viscosity of glasses. Journal of the American Ceramic Society 8, 339-355.

Gainaru, C., Kastner, S., Mayr, F., Lunkenheimer, P., Schildmann, S., Weber, H., Hiller, W., Loidl, A., Böhmer, R., 2011. Hydrogen-bond equilibria and lifetimes in a monohydroxy alcohol. Physical Review Letters 107, 118304.

Ge, Q., Luo, X., Iversen, C.B., Mather, P.T., Dunn, M.L., Qi, H.J., 2013. Mechanisms of tripleshape polymeric composites due to dual thermal transitions. Soft Matter 9, 2212-2223.

Ge, Q., Luo, X., Iversen, C.B., Nejad, H.B., Mather, P.T., Dunn, M.L., Qi, H.J., 2014. A finite deformation thermomechanical constitutive model for triple shape polymeric composites based on dual thermal transitions. International Journal of Solids and Structures 51, 2777-2790.

Ge, Q., Yu, K., Ding, Y., Qi, H.J., 2012. Prediction of temperature-dependent free recovery behaviors of amorphous shape memory polymers. Soft Matter 8, 11098-11105.

Ghosh, P., Srinivasa, A., 2014. Development of a finite strain two-network model for shape memory polymers using QR decomposition. International Journal of Engineering Science 81, 177-191. 
Gibbs, J.H., DiMarzio, E.A., 1958. Nature of the glass transition and the glassy state. The Journal of Chemical Physics 28, 373-383.

Gilormini, P., Diani, J., 2012. On modeling shape memory polymers as thermoelastic two-phase composite materials. Comptes Rendus Mécanique 340, 338-348.

Glotzer, S.C., 2000. Spatially heterogeneous dynamics in liquids: insights from simulation. Journal of Non-Crystalline Solids 274, 342-355.

Guo, X., Liu, L., Zhou, B., Liu, Y., Leng, J., 2015. Constitutive model for shape memory polymer based on the viscoelasticity and phase transition theories. Journal of Intelligent Material Systems and Structures, doi: 1045389X15571380.

Gupta, P.K., Mauro, J.C., 2007. The laboratory glass transition. The Journal of Chemical Physics 126, 224504.

Hassan, T., Taleb, L., Krishna, S., 2008. Influence of non-proportional loading on ratcheting responses and simulations by two recent cyclic plasticity models. International Journal of Plasticity 24, 1863-1889.

Hill, R., 1965. A self-consistent mechanics of composite materials. Journal of the Mechanics and Physics of Solids 13, 213-222.

Hitov, J.J., Rainer, W.C., Redding, E.M., Sloan, A.W., Stewart, W.D., 1964. Polyethylene product and process. Google Patents.

Hori, M., Nemat-Nasser, S., 1993. Double-inclusion model and overall moduli of multi-phase composites. Mechanics of Materials 14, 189-206.

Hu, G., Weng, G., 2000. Some reflections on the Mori-Tanaka and Ponte Castaneda-Willis methods with randomly oriented ellipsoidal inclusions. Acta Mechanica 140, 31-40.

Hu, J., Zhu, Y., Huang, H., Lu, J., 2012. Recent advances in shape-memory polymers: Structure, mechanism, functionality, modeling and applications. Progress in Polymer Science 37, 17201763.

Huang, W., Ding, Z., Wang, C., Wei, J., Zhao, Y., Purnawali, H., 2010. Shape memory materials. Materials Today 13, 54-61.

Huang, W., Yang, B., An, L., Li, C., Chan, Y., 2005. Water-driven programmable polyurethane shape memory polymer: demonstration and mechanism. Applied Physics Letters 86, 114105.

Ivens, J., Urbanus, M., De Smet, C., 2011. Shape recovery in a thermoset shape memory polymer and its fabric-reinforced composites. eXPRESS Polymer Letters 5, 254-261.

Ju, J., Chen, T., 1994. Effective elastic moduli of two-phase composites containing randomly dispersed spherical inhomogeneities. Acta Mechanica 103, 123-144.

Kafka, V., 2008. Shape memory polymers: a mesoscale model of the internal mechanism leading to the SM phenomena. International Journal of Plasticity 24, 1533-1548.

Kauzmann, W., 1948. The Nature of the Glassy State and the Behavior of Liquids at Low Temperatures. Chemical Reviews 43, 219-256.

Kazakevičiūtė-Makovska, R., Steeb, H., Aydin, A.Ö., 2012. On the evolution law for the frozen fraction in linear theories of shape memory polymers. Archive of Applied Mechanics 82, 1103 1115.

Kim, J.H., Kang, T.J., Yu, W.-R., 2010. Thermo-mechanical constitutive modeling of shape memory polyurethanes using a phenomenological approach. International Journal of Plasticity 26, 204-218.

Kudlik, A., Benkhof, S., Blochowicz, T., Tschirwitz, C., Rössler, E., 1999. The dielectric response of simple organic glass formers. Journal of Molecular Structure 479, 201-218.

Lendlein, A., Jiang, H., Jünger, O., Langer, R., 2005. Light-induced shape-memory polymers. Nature 434, 879-882.

Lendlein, A., Kelch, S., 2002. Shape memory polymers. Angewandte Chemie International Edition 41, 2034-2057.

Leng, J., Lan, X., Liu, Y., Du, S., 2011. Shape-memory polymers and their composites: stimulus methods and applications. Progress in Materials Science 56, 1077-1135. 
Leng, J., Lu, H., Liu, Y., Huang, W.M., Du, S., 2009. Shape-memory polymers - a class of novel smart materials. MRS Bulletin 34, 848-855.

Levin, V., 1967. Thermal expansion coefficients of heterogeneous materials. Mekhanika Tverdogo Tela 2, 88-94.

Levitas, V.I., Ozsoy, I.B., 2009a. Micromechanical modeling of stress-induced phase transformations. Part 1. Thermodynamics and kinetics of coupled interface propagation and reorientation. International Journal of Plasticity 25, 239-280.

Levitas, V.I., Ozsoy, I.B., 2009b. Micromechanical modeling of stress-induced phase transformations. Part 2. Computational algorithms and examples. International Journal of Plasticity 25, 546-583.

Li, G., 2014. Self-Healing Composites: Shape Memory Polymer Based Structures. John Wiley \& Sons, Inc., West Sussex, UK.

Li, G., Ajisafe, O., Meng, H., (2013). Effect of Strain Hardening of Shape Memory Polymer Fibers on Healing Efficiency of Thermosetting Polymer Composites. Polymer 54, 920-928.

Li, G., Lee-Sullivan, P., Thring, R., 2000. Determination of activation energy for glass transition of an epoxy adhesive using dynamic mechanical analysis. Journal of Thermal Analysis and Calorimetry 60, 377-390.

Li, G., Nettles, D., 2010. Thermomechanical characterization of a shape memory polymer based self-repairing syntactic foam. Polymer 51, 755-762.

Li, G., Shojaei, A., 2012. A viscoplastic theory of shape memory polymer fibres with application to self-healing materials. Proceedings of the Royal Society A: Mathematical, Physical and Engineering Science 468, 2319-2346.

Li, G., Uppu, N., 2010. Shape memory polymer based self-healing syntactic foam: 3-D confined thermomechanical characterization. Composites Science and Technology 70, 1419-1427.

Li, G., Xu, W., 2011. Thermomechanical behavior of thermoset shape memory polymer programmed by cold-compression: testing and constitutive modeling. Journal of the Mechanics and Physics of Solids 59, 1231-1250.

Li, G., Zhang, P, (2013). A Self-healing Particulate Composite Reinforced with Strain Hardened Short Shape Memory Polymer Fibers. Polymer 54, 5075-5086.

Li, L., Wang, T., 2005. A unified approach to predict overall properties of composite materials. Materials Characterization 54, 49-62.

Lin, J., Chen, L., 1999. Shape-memorized crosslinked ester - type polyurethane and its mechanical viscoelastic model. Journal of Applied Polymer Science 73, 1305-1319.

Liu, C., Qin, H., Mather, P., 2007. Review of progress in shape-memory polymers. Journal of Materials Chemistry 17, 1543-1558.

Liu, S., Jiao, W., Sun, B., Wang, W., 2013. A quasi-phase perspective on flow units of glass transition and plastic flow in metallic glasses. Journal of Non-Crystalline Solids 376, 76-80.

Liu, Y., Gall, K., Dunn, M.L., Greenberg, A.R., Diani, J., 2006. Thermomechanics of shape memory polymers: uniaxial experiments and constitutive modeling. International Journal of Plasticity 22, 279-313.

Long, K.N., Dunn, M.L., Qi, H.J., 2010. Mechanics of soft active materials with phase evolution. International Journal of Plasticity 26, 603-616.

Long, K.N., Scott, T.F., Qi, H.J., Bowman, C.N., Dunn, M.L., 2009. Photomechanics of lightactivated polymers. Journal of the Mechanics and Physics of Solids 57, 1103-1121.

Lunkenheimer, P., Kastner, S., Köhler, M., Loidl, A., 2010. Temperature development of glassy $\alpha-$ relaxation dynamics determined by broadband dielectric spectroscopy. Physical Review E 81, 051504.

Mather, P.T., Luo, X., Rousseau, I.A., 2009. Shape memory polymer research. Annual Review of Materials Research 39, 445-471.

Messé, L., Pézolet, M., Prud'homme, R., 2001. Molecular relaxation study of polystyrene: influence of temperature, draw rate and molecular weight. Polymer 42, 563-575. 
Meng, H., Li, G., 2013. A review of stimuli-responsive shape memory polymer composites. Polymer 54, 2199-2221.

Mori, T., Tanaka, K., 1973. Average stress in matrix and average elastic energy of materials with misfitting inclusions. Acta Metallurgica 21, 571-574.

Moumni, Z., Zaki, W., Nguyen, Q.S., 2008. Theoretical and numerical modeling of solid-solid phase change: application to the description of the thermomechanical behavior of shape memory alloys. International Journal of Plasticity 24, 614-645.

Müller, C., Bruhns, O., 2006. A thermodynamic finite-strain model for pseudoelastic shape memory alloys. International Journal of Plasticity 22, 1658-1682.

Nettles, D., 2009. Thermechanical Charaterization of A Shape Memory Polymer Based Syntactioc Foam. M.S. Thesis, Louisiana State University.

Nguyen, T., Yakacki, C.M., Brahmbhatt, P.D., Chambers, M.L., 2010. Modeling the relaxation mechanisms of amorphous shape memory polymers. Advanced Materials 22, 3411-3423.

Nguyen, T.D., Jerry Qi, H., Castro, F., Long, K.N., 2008. A thermoviscoelastic model for amorphous shape memory polymers: incorporating structural and stress relaxation. Journal of the Mechanics and Physics of Solids 56, 2792-2814.

Paluch, M., 2000. Dielectric and mechanical relaxation in epoxy systems with molecules of differing topology. Journal of Physics: Condensed Matter 12, 9511.

Patashinski, A., 2014. Stress-induced glass transitions. International Journal of Engineering Science 83, 95-98.

Peng, X., Hu, N., Zheng, H., Fukunaga, H., 2009. Evaluation of mechanical properties of particulate composites with a combined self-consistent and Mori-Tanaka approach. Mechanics of Materials 41, 1288-1297.

Pieczyska, E., Maj, M., Kowalczyk-Gajewska, K., Staszczak, M., Gradys, A., Majewski, M., Cristea, M., Tobushi, H., Hayashi, S., 2015. Thermomechanical properties of polyurethane shape memory polymer-experiment and modelling. Smart Materials and Structures 24, 045043.

Popov, P., Lagoudas, D.C., 2007. A 3-D constitutive model for shape memory alloys incorporating pseudoelasticity and detwinning of self-accommodated martensite. International Journal of Plasticity 23, 1679-1720.

Porter, D., 1995. Group interaction modelling of polymer properties, first ed. Marcel Dekke, New York.

Qi, H.J., Nguyen, T.D., Castro, F., Yakacki, C.M., Shandas, R., 2008. Finite deformation thermomechanical behavior of thermally induced shape memory polymers. Journal of the Mechanics and Physics of Solids 56, 1730-1751.

Ratna, D., Karger-Kocsis, J., 2008. Recent advances in shape memory polymers and composites: a review. Journal of Materials Science 43, 254-269.

Reese, S., Böl, M., Christ, D., 2010. Finite element-based multi-phase modelling of shape memory polymer stents. Computer Methods in Applied Mechanics and Engineering 199, 1276-1286.

Reese, S., Christ, D., 2008. Finite deformation pseudo-elasticity of shape memory alloysconstitutive modelling and finite element implementation. International Journal of Plasticity 24, 455-482.

Reinsberg, S., Qiu, X., Wilhelm, M., Spiess, H.W., Ediger, M., 2001. Length scale of dynamic heterogeneity in supercooled glycerol near Tg. The Journal of Chemical Physics 114, 72997302.

Richert, R., 2002. Heterogeneous dynamics in liquids: fluctuations in space and time. Journal of Physics: Condensed Matter 14, R703.

Rosen, B.W., Hashin, Z., 1970. Effective thermal expansion coefficients and specific heats of composite materials. International Journal of Engineering Science 8, 157-173.

Sasai, M., 2003. Energy landscape picture of supercooled liquids: Application of a generalized random energy model. The Journal of Chemical Physics 118, 10651-10662. 
Scalet, G., Auricchio, F., Bonetti, E., Castellani, L., Ferri, D., Pachera, M., Scavello, F., 2015. An experimental, theoretical and numerical investigation of shape memory polymers. International Journal of Plasticity 67, 127-147.

Schmidt, A.M., 2006. Electromagnetic activation of shape memory polymer networks containing magnetic nanoparticles. Macromolecular Rapid Communications 27, 1168-1172.

Schönhals, A., 1998. Dielectric spectroscopy onthe dynamics of amorphous polymeric systems. Application note Dielectrics 1.

Scott, T.F., Schneider, A.D., Cook, W.D., Bowman, C.N., 2005. Photoinduced plasticity in crosslinked polymers. Science 308, 1615-1617.

Sharafi, S., Li, G., 2015. A multiscale approach for modeling actuation response of polymeric artificial muscles. Soft Matter 11, 3833 - 3843.

Shell, M.S., Debenedetti, P.G., Stillinger, F.H., 2005. Dynamic heterogeneity and non-Gaussian behaviour in a model supercooled liquid. Journal of Physics: Condensed Matter 17, S4035.

Shojaei, A., Li, G., 2013. Viscoplasticity analysis of semicrystalline polymers: a multiscale approach within micromechanics framework. International Journal of Plasticity 42, 31-49.

Shojaei, A., Li, G., 2014. Thermomechanical constitutive modelling of shape memory polymer including continuum functional and mechanical damage effects. Proceedings of the Royal Society A: Mathematical, Physical and Engineering Science 470, 20140199.

Strobl, G.R., 2007. The Physics of Polymers: Concepts for Understanding Their Structures and Behavior, third ed. Springer .

Tammann, G., Hesse, W., 1926. The dependence of viscosity upon the temperature of supercooled liquids. Zeitschrift für anorganische und allgemeine Chemie 156, 245-257.

Thamburaja, P., Ekambaram, R., 2007. Coupled thermo-mechanical modelling of bulk-metallic glasses: theory, finite-element simulations and experimental verification. Journal of the Mechanics and Physics of Solids 55, 1236-1273.

Thamburaja, P., Nikabdullah, N., 2009. A macroscopic constitutive model for shape-memory alloys: theory and finite-element simulations. Computer Methods in Applied Mechanics and Engineering 198, 1074-1086.

Thirtha, V., Lehman, R., Nosker, T., 2006. Morphological effects on glass transition behavior in selected immiscible blends of amorphous and semicrystalline polymers. Polymer 47, 53925401.

Tobolsky, A., Eyring, H., 1943. Mechanical properties of polymeric materials. The Journal of Chemical Physics 11, 125-134.

Tobushi, H., Hashimoto, T., Hayashi, S., Yamada, E., 1997. Thermomechanical constitutive modeling in shape memory polymer of polyurethane series. Journal of Intelligent Material Systems and Structures 8, 711-718.

Tobushi, H., Okumura, K., Hayashi, S., Ito, N., 2001. Thermomechanical constitutive model of shape memory polymer. Mechanics of Materials 33, 545-554.

Véchambre, C., Buléon, A., Chaunier, L., Gauthier, C., Lourdin, D., 2011. Understanding the mechanisms involved in shape memory starch: macromolecular orientation, stress recovery and molecular mobility. Macromolecules 44, 9384-9389.

Vernon, L.B., Vernon, H.M., 1941. Process of manufacturing articles of thermoplastic synthetic resins. Google Patents.

Vogel, H., 1921. The law of viscosity change with temperature. Physikalische Zeitschrift 22, 645646.

Volk, B.L., Lagoudas, D.C., Maitland, D.J., 2011. Characterizing and modeling the free recovery and constrained recovery behavior of a polyurethane shape memory polymer. Smart Materials and Structures 20, 094004.

Vyazovkin, S., Sbirrazzuoli, N., Dranca, I., 2006. Variation in activation energy of the glass transition for polymers of different dynamic fragility. Macromolecular Chemistry and Physics 207, 1126-1130. 
Wales, D., 2004. Energy Landscapes: Applications to Clusters, Biomolecules and Glasses, first ed. Cambridge University Press, Cambridge.

Wang, A., Li, G., 2015. Stress memory of a thermoset shape memory polymer. Journal of Applied Polymer Science 132, 42112.

Wang, X., Xu, B., Yue, Z., 2008. Micromechanical modelling of the effect of plastic deformation on the mechanical behaviour in pseudoelastic shape memory alloys. International Journal of Plasticity 24, 1307-1332.

Wang, Z., Li, D., Xiong, Z., Chang, R., 2009. Modeling thermomechanical behaviors of shape memory polymer. Journal of Applied Polymer Science 113, 651-656.

Weng, G., 1984. Some elastic properties of reinforced solids, with special reference to isotropic ones containing spherical inclusions. International Journal of Engineering Science 22, 845-856.

Westbrook, K.K., Kao, P.H., Castro, F., Ding, Y., Qi, H.J., 2011. A 3D finite deformation constitutive model for amorphous shape memory polymers: a multi-branch modeling approach for nonequilibrium relaxation processes. Mechanics of Materials 43, 853-869.

Westbrook, K.K., Parakh, V., Chung, T., Mather, P.T., Wan, L.C., Dunn, M.L., Qi, H.J., 2010. Constitutive modeling of shape memory effects in semicrystalline polymers with stretch induced crystallization. Journal of Engineering Materials and Technology 132, 041010.

Wu, S., 1989. Surface and Interfacial Tensions of Polymers, Oligomers, Plasticizers, and Organic Pigments, in: J. Brandrup, Immergut, E.H. (Eds.), Polymer Handbook third ed. WileyInterscience, New York, 414-426.

Xiao, R., Choi, J., Lakhera, N., Yakacki, C.M., Frick, C.P., Nguyen, T.D., 2013. Modeling the glass transition of amorphous networks for shape-memory behavior. Journal of the Mechanics and Physics of Solids 61, 1612-1635.

Xie, T., 2010. Tunable polymer multi-shape memory effect. Nature 464, 267-270.

$\mathrm{Xu}, \mathrm{W}$., Li, G., 2010. Constitutive modeling of shape memory polymer based self-healing syntactic foam. International Journal of Solids and Structures 47, 1306-1316.

$\mathrm{Xu}, \mathrm{W} ., \mathrm{Li}, \mathrm{G} ., 2$ 2011. Thermoviscoplastic modeling and testing of shape memory polymer based self-healing syntactic foam programmed at glassy temperature. Journal of Applied MechanicsTransactions of ASME 78, 061017.

Yang, Q., Li, G., 2014a. Investigation into stress recovery behavior of shape memory polyurethane fiber. Journal of Polymer Science Part B: Polymer Physics 52, 1429-1440.

Yang, Q., Li, G., 2014b. Spider-silk-like shape memory polymer fiber for vibration damping. Smart Materials and Structures 23, 105032.

Yu, K., Ge, Q., Qi, H.J., 2014. Reduced time as a unified parameter determining fixity and free recovery of shape memory polymers. Nature communications 5, doi:10.1038/ncomms 4066 .

Yu, K., Xie, T., Leng, J., Ding, Y., Qi, H.J., 2012. Mechanisms of multi-shape memory effects and associated energy release in shape memory polymers. Soft Matter 8, 5687-5695.

Zhang, C., Hu, J., Chen, S., Ji, F., 2010. Theoretical study of hydrogen bonding interactions on MDI-based polyurethane. Journal of Molecular Modeling 16, 1391-1399.

Zhao, Q., Qi, H.J., Xie, T., 2015. Recent Progress in Shape Memory Polymer: New Behavior, Enabling Materials, and Mechanistic Understanding. Progress in Polymer Science, doi:10.1016/j.progpolymsci.2015.04.001

Zhou, Z., Chudnovsky, A., Bosnyak, C., Sehanobish, K., 1995. Cold-drawing (necking) behavior of polycarbonate as a double glass transition. Polymer Engineering \& Science 35, 304-309.

Zwanzig, R., 2001. Nonequilibrium statistical mechanics, first ed. Oxford University Press, Oxford. 